\title{
Laminar-turbulent transition induced by a discrete roughness element in a supersonic boundary layer
}

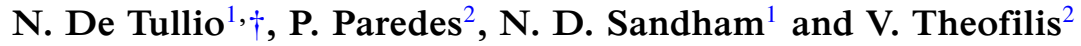 \\ ${ }^{1}$ Aeronautics and Astronautics, Faculty of Engineering and the Environment, \\ University of Southampton, Southampton SO17 1BJ, UK \\ ${ }^{2}$ School of Aeronautics, Universidad Politécnica de Madrid, Plaza Cardenal Cisneros 3, \\ E-28040 Madrid, Spain
}

(Received 17 April 2013; revised 25 August 2013; accepted 26 September 2013)

The linear instability and breakdown to turbulence induced by an isolated roughness element in a boundary layer at Mach 2.5, over an isothermal flat plate with laminar adiabatic wall temperature, have been analysed by means of direct numerical simulations, aided by spatial BiGlobal and three-dimensional parabolized (PSE-3D) stability analyses. It is important to understand transition in this flow regime since the process can be slower than in incompressible flow and is crucial to prediction of local heat loads on next-generation flight vehicles. The results show that the roughness element, with a height of the order of the boundary layer displacement thickness, generates a highly unstable wake, which is composed of a low-velocity streak surrounded by a three-dimensional high-shear layer and is able to sustain the rapid growth of a number of instability modes. The most unstable of these modes are associated with varicose or sinuous deformations of the low-velocity streak; they are a consequence of the instability developing in the three-dimensional shear layer as a whole (the varicose mode) or in the lateral shear layers (the sinuous mode). The most unstable wake mode is of the varicose type and grows on average $\sim 17 \%$ faster than the most unstable sinuous mode and $\sim 30$ times faster than the most unstable boundary layer mode occurring in the absence of a roughness element. Due to the high growthrates registered in the presence of the roughness element, an amplification factor of $N=9$ is reached within $\sim 50$ roughness heights from the roughness trailing edge. The independently performed Navier-Stokes, spatial BiGlobal and PSE-3D stability results are in excellent agreement with each other, validating the use of simplified theories for roughness-induced transition involving wake instabilities. Following the linear stages of the laminar-turbulent transition process, the roll-up of the three-dimensional shear layer leads to the formation of a wedge of turbulence, which spreads laterally at a rate similar to that observed in the case of compressible turbulent spots for the same Mach number.

Key words: compressible boundary layers, instability, transition to turbulence

$\dagger$ Present address: Department of Mathematics, Imperial College London, South Kensington Campus, London SW7 2AZ, UK. Email address for correspondence: n.de-tullio@imperial.ac.uk 


\section{Introduction}

Experimental observations indicate that the dominant laminar-turbulent transition mechanisms in boundary layers can be greatly modified by the presence of localized or distributed roughness, leading to a significant acceleration of the transition process both in noisy and in quiet environments (Tani \& Sato 1956; Klebanoff \& Tidstrom 1972; Corke, Bar-Server \& Morkovin 1986; Fujii 2006; Schneider 2008). Over the years, a number of possible scenarios have been postulated to explain these observations. However, despite numerous research efforts, the underlying physical mechanisms responsible for roughness-induced transition are only partly understood. Consequently high-speed vehicle design still relies on empirical transition-prediction criteria (Reda 2002). A deeper understanding of the effects of roughness on the stability of boundary layers is thus urgently needed since it is particularly important for high-speed applications, where transition to turbulence leads to a significant increase of both the skin friction and the wall heat transfer, with obvious implications for the design of high-speed vehicles.

The early experiments of Klebanoff \& Tidstrom (1972) showed that discrete twodimensional roughness elements may lead to an acceleration of the transition process due to the modified stability of the tripped boundary layer. In particular, the growth of Tollmien-Schlichting (TS) waves was found to be enhanced by the two-dimensional roughness element. The effects of localized two-dimensional roughness elements on the stability of a compressible boundary layer at $M=4.5$ have recently been studied via direct numerical simulation (DNS) by Marxen, Iaccarino \& Shaqfeh (2010), who showed that the roughness element induces a sudden disturbance amplification (over a limited frequency band) in its vicinity but does not affect the boundary layer stability characteristics further downstream. The sudden amplification appears to be due to the phase-locked linear superposition of the first boundary layer mode with a stable mode excited by the roughness element, resulting in transient energy growth. This result seems to be in line with a mechanism, originally proposed by Ruban (1984) and Goldstein (1985) and studied theoretically by Choudhari \& Street (1992) and Crouch (1992), by which the interaction of free-stream disturbances with localized surface geometrical distortions can excite disturbances 'tuned' (in frequency and phase speed) with the boundary layer instability eigenmodes. An example of this mechanism for three-dimensional localized roughness elements, in the context of compressible boundary layers, was reported in the DNS results of Balakumar (2008). However, the effect of three-dimensional roughness elements on the stability of boundary layers seems to extend beyond the enhancement of the receptivity, that is, conversion of external disturbance in boundary layer modes, and/or amplification of boundary layer modes. Three-dimensional roughness elements often induce subcritical disturbance amplification, leading to a 'bypass'-like laminar-turbulent transition. In fact, bypass and roughness-induced transition to turbulence appear to share numerous similarities, at least in the incompressible flow regime.

Bypass transition may take place when moderate or large free-stream disturbances enter the boundary layer and give rise to high- and low-velocity streaks through the 'lift-up' mechanism of Landahl (1975) (see also Hultgren \& Gustavsson 1981; Alfredsson \& Matsubara 1996; Andersson, Berggren \& Henningson 1999; Jacobs \& Durbin 2001; Reddy et al. 2001; Reshotko 2001). The streaks may then interact nonlinearly with each other (Brandt \& de Lange 2008) or undergo a secondary instability before breaking down into turbulence (Andersson et al. 2001). Investigations into the secondary instability of streaks in boundary layers have been conducted 
mainly for incompressible flows. The early experiments of Swearingen \& Blackwelder (1987) show that low-velocity streaks, appearing following the formation of Görtler vortices in a concave wall boundary layer, can sustain both sinuous (anti-symmetric) and varicose (symmetric) instabilities, the former being more prominent. More recently Asai, Minagawa \& Nishioka (2002) experimentally investigated the linear instability of a single boundary layer streak, showing that under certain conditions (mainly depending on the geometry of the three-dimensional high-shear layer surrounding the streak) the varicose mode can be more unstable than the sinuous mode. In the nonlinear transition stages, quasi-streamwise vortices and hairpin vortices form following a sinuous and a varicose streak instability, respectively. The growth of the varicose mode was found to be the consequence of a Kelvin-Helmholtz instability of the wall-normal detached high-shear layer, while the sinuous mode develops due to an instability of the lateral high-shear layers. Numerical simulations performed by Andersson et al. (2001) indicate that the streak instability starts appearing in the form of a sinuous perturbation for streak amplitudes of $\sim 26 \%$ of the free-stream velocity, while varicose disturbances become unstable only for streak amplitudes larger than $37 \%$. Their inviscid stability simulations indicate that the sinuous mode grows faster than the varicose instability; a result which was also reported by $\mathrm{Wu} \&$ Choudhari (2003).

Roughness-induced transition may follow a similar path. In fact, the flow behind small three-dimensional localized roughness elements is characterized by the presence of counter-rotating streamwise vortices (see for example Gaster, Grosch \& Jackson 1994; Joslin \& Grosch 1995; Tumin \& Reshotko 2005; Rizzetta \& Visbal 2007), which through the 'lift-up' effect can potentially lead to strong algebraic growth of boundary layer streaks and breakdown to turbulence. A model based on the transient growth mechanism was proposed by Reshotko \& Tumin (2004) to predict roughnessinduced transition, following a similar approach to that of Andersson et al. (2001) for the bypass transition scenario. The model suggests that the transition Reynolds number (based on the momentum thickness $\theta)$ varies according to $(h / \theta)^{-1}$ for stagnation point flow with constant wall temperature, thereby agreeing with the ballistic-range data presented by Reda (2002). Reda's review provides a detailed analysis of the available experimental data for a variety of different flows and provides strong evidence of the importance of the critical roughness Reynolds number approach $\left(R e_{h}=u_{h} h / v_{h}\right.$, where $u_{h}$ and $v_{h}$ are the streamwise velocity and kinematic viscosity taken at $y=h$ in the corresponding clean flat-plate boundary layer) for modelling laminar-turbulent transition induced by localized and distributed roughness. However, Reda also concludes that a universal critical $R e_{h}$ will not be found, given the large number of different flow conditions and roughness patterns possible. Somewhat in contrast with this view, Horvath, Berri \& Merski (2004) propose a transition criterion for isolated roughness elements based on the parameter momentum thickness Reynolds number divided by the boundary layer edge Mach number. Critical values of this parameter appear to scale linearly (on a logarithmic scale) with the roughness height divided by the local boundary layer thickness.

A parametric study of the effects of localized smooth roughness elements on the laminar-turbulent transition occurring in supersonic flat-plate boundary layers was recently performed by Redford, Sandham \& Roberts (2010) using DNS. Their results indicate that transition is promoted provided $R e_{h}$ exceeds a critical value which increases as the parameter $M_{h} T_{\infty} / T_{w}$ increases, where $M_{h}$ is the Mach number calculated at the roughness edge and $T_{w}$ is the wall temperature. They proposed a roughness-induced transition map which suggests a critical value of $R e_{h}=300$ 
for $M_{h} T_{\infty} / T_{w}=0$. These findings were confirmed by Bernardini, Pirozzoli \& Orlandi (2012), who proposed a modified version of roughness Reynolds number $R e_{h}^{*}=U_{h} h / v_{w}$, with the kinematic viscosity taken at the wall, which has a constant critical value of $R e_{h}^{*}=460$.

The criteria mentioned above represent a useful tool for predicting roughnessinduced transition, without attempting to provide a physical explanation of the flow instability and transition to turbulence. In order to gain a better understanding of the physical mechanisms driving the roughness-induced transition process, Choudhari and co-workers (Choudhari et al. 2010; Kegerise et al. 2012) analysed the growth of instabilities in the wake of diamond-shaped roughness elements at $M=3.5$, both experimentally and through BiGlobal linear stability calculations. The results show that both sinuous and varicose modes can grow substantially in the linear stages of the transition process. For $R e_{h}=426$ the experiments show that the transition process is driven by the varicose mode, while the sinuous mode dominates for $R e_{h}=319$. This result is in agreement with the BiGlobal stability simulations, which show that the sinuous mode becomes more dominant for decreasing $R e_{h}$. The BiGlobal stability results also suggest that the varicose mode growth-rate decreases more rapidly than that of the sinuous mode with increasing streamwise distance, suggesting that the latter mode may drive the final breakdown to turbulence even for high $R e_{h}$. Experimentally obtained mode shapes were found to be in good qualitative agreement with the eigenfunctions extracted from the BiGlobal stability analysis. Groskopf, Kloker \& Marxen (2009) studied the instability induced by discrete three-dimensional roughness elements in an $M=4.8$ boundary layer, reporting good agreement between BiGlobal stability simulations and DNS. Further experimental results demonstrating the importance of the roughness wake instability as the cause of the early laminar-turbulent transition observed in the presence of isolated roughness elements were reported by Kegerise, Owens \& Rudolf (2010) and Owens, Kegerise \& Wilkinson (2011). Recently Wheaton \& Schneider $(2012$, 2013) have carried out a set of experiments on roughness-induced transition at $M=6$, reporting the first quantitative measurements of the roughness wake instability at hypersonic speeds. For the same Mach number, the numerical simulations of De Tullio \& Sandham (2012) and Choudhari et al. (2013) show that the roughness wake can sustain the growth of a number of different instability modes, the relative importance of which seems to depend on the specific flow conditions considered.

In the wake flow behind an isolated three-dimensional roughness element, a predominant spatial direction exists along which the mean flow properties vary slowly, while the flow varies rapidly in the cross-flow directions. These properties are taken into account in the derivation of the three-dimensional parabolized stability equations (PSE-3D), which represent the most efficient simplified approach for the solution of the instability problem of such flows. In an analogous manner to the extension of the Orr-Sommerfeld and Squire equations to the conventional PSE, the BiGlobal analysis equations are extended to the PSE-3D analysis. The linear PSE-3D methodology, formulated in the incompressible limit in Galionis \& Hall (2005) (see also Broadhurst, Theofilis \& Sherwin 2006; Broadhurst \& Sherwin 2008; Paredes et al. 2011) and for the first time in the compressible flow regime here, assumes the existence of two scales along the streamwise spatial direction: a slow one, on which the base state varies, and a fast one, along which linear and nonlinear wave-like perturbations develop. Base flow and disturbances are inhomogeneous functions of the two coordinates of the cross-sectional planes, and hence the two directions need to be resolved in a coupled manner. Therefore, the PSE-3D methodology resolves a 
PDE-based instability problem at each streamwise location. After initializing the PSE$3 \mathrm{D}$ with solutions of the spatial BiGlobal eigenvalue problem at a given cross-section of the geometry analysed (Paredes et al. 2011), the full three-dimensional disturbance equations are marched along the slowly varying spatial direction, solving the aforementioned PDE-based stability problem (with two-dimensional eigenfunctions) at each station. It should be noted that the term PSE-3D has been used in the literature to describe the standard PSE (Herbert 1997) as applied to three-dimensional boundary layers (as in Mughal 2006). Here, the term PSE-3D is redefined in the sense of Broadhurst et al. (2006) and Paredes et al. (2011) to denote an instability analysis methodology in which the base flow is strongly dependent on two spatial directions.

In this work we study the linear and nonlinear stages of the laminar-turbulent transition process induced by a sharp-edged isolated roughness element embedded in a Mach 2.5 boundary layer. This Mach number is high enough for significant compressibility effects to be expected but below the Mach number at which higher modes of instability (Mack modes) develop. The linear instability of the flow is investigated through full Navier-Stokes (NS) simulations, spatial BiGlobal and linear PSE-3D stability analyses. The breakdown to turbulence following the linear growth of instability modes is investigated by DNS. The remainder of the paper is organized as follows. In $\S 2$ we present the Navier-Stokes, BiGlobal stability and PSE-3D equations, while the numerics used for their solution are described in $\S 3$, along with details of the numerical simulations carried out. A discussion of the results obtained is provided in $\S 4$ and the paper ends with some conclusions in $\S 5$.

\section{Problem formulation}

\subsection{Navier-Stokes equations}

The equations governing the motion of a Newtonian fluid with viscosity $\mu$ are obtained by imposing conservation of mass, momentum and energy. The result is a system of nonlinear partial differential equations (PDEs) which in dimensionless form can be written as

$$
\begin{gathered}
\frac{\partial \rho}{\partial t}+\frac{\partial \rho u_{j}}{\partial x_{j}}=0 \\
\frac{\partial \rho u_{i}}{\partial t}+\frac{\partial \rho u_{i} u_{j}}{\partial x_{j}}+\frac{\partial p}{\partial x_{i}}=\frac{\partial \tau_{i j}}{\partial x_{j}} \\
\frac{\partial \rho E}{\partial t}+\frac{\partial(\rho E+p) u_{i}}{\partial x_{i}}=-\frac{\partial q_{i}}{\partial x_{i}}+\frac{\partial u_{i} \tau_{i j}}{\partial x_{j}} .
\end{gathered}
$$

The symmetric viscous stress tensor $\tau$ is defined by

$$
\tau_{i j}=\frac{\mu}{R e}\left(\frac{\partial u_{j}}{\partial x_{i}}+\frac{\partial u_{i}}{\partial x_{j}}-\frac{2}{3} \frac{\partial u_{k}}{\partial x_{k}} \delta_{i j}\right),
$$

where $\delta_{i j}$ is the Kronecker delta defined by $\delta_{i j}=1$ for $i=j$ and $\delta_{i j}=0$ for $i \neq j$. The properties of the fluid and the components of the heat flux vector $\left(\boldsymbol{q}_{j}\right)$ are calculated considering the equation of state and Fourier's law of heat conduction, given respectively by

$$
p=(\gamma-1)\left(\rho E-\frac{1}{2} \rho u_{i} u_{i}\right)=\frac{1}{\gamma M^{2}} \rho T \quad \text { and } \quad q_{j}=-\frac{\mu}{(\gamma-1) M^{2} \operatorname{Pr} \operatorname{Re}} \frac{\partial T}{\partial x_{j}} .
$$


Sutherland's law, $\mu=T^{3 / 2}\left(1+S^{*} / T_{r}^{*}\right) /\left(T+S^{*} / T_{r}^{*}\right)$, is used for the calculation of the dynamic viscosity, where $S^{*}=110.4 \mathrm{~K}$ is the Sutherland constant for air and $T_{r}^{*}=273.15 \mathrm{~K}$. Dimensionless parameters which define the problem are the Reynolds number $R e$, the Mach number $M$ and the Prandtl number $P r$, which is considered to be constant and equal to $\operatorname{Pr}=0.72$. Note that the subscript $(r)$ refers to reference values whereas the asterisks $\left(^{*}\right)$ denote dimensional variables. The reference values for velocity $\left(u_{r}^{*}\right)$, density $\left(\rho_{r}^{*}\right)$, temperature $\left(T_{r}^{*}\right)$ and dynamic viscosity $\left(\mu_{r}^{*}\right)$ are taken at the free stream, while the reference length $\left(l_{r}^{*}\right)$ is taken as the displacement thickness of the laminar boundary layer at the computational domain inflow (more details in $\S 3$ ).

\subsection{Linear instability analyses}

The analysis of flow stability used here is also based on the compressible equations of motion, and monitors linear modal perturbation development. The development in time and space of small-amplitude perturbations superposed upon a given flow can be described by the linearized Navier-Stokes equations. Linearization of the equations of motion is performed around a laminar steady flow, denoted as "base flow', $\boldsymbol{q}_{b}=\left(\rho_{b}, u_{b}, v_{b}, w_{b}, T_{b}\right)^{\mathrm{T}}$.

In using the term 'small-amplitude perturbations', solutions to the initial-valueproblem

$$
\boldsymbol{B}\left(R e, M, \boldsymbol{q}_{b}\right) \frac{\mathrm{d} \tilde{\boldsymbol{q}}}{\mathrm{d} t}=\boldsymbol{A}\left(R e, M, \boldsymbol{q}_{b}\right) \tilde{\boldsymbol{q}},
$$

are considered, where $\tilde{\boldsymbol{q}}(x, y, z, t)=(\tilde{\rho}, \tilde{u}, \tilde{v}, \tilde{w}, \tilde{T})^{\mathrm{T}}$ is the vector comprising the linear density, velocity components and temperature perturbations; see Theofilis (2011) for more details.

\subsubsection{The PSE-3D equations}

The PSE-3D equations are valid for convectively unstable flows in which the disturbance root mean square values vary slowly in the streamwise direction, i.e. $\partial / \partial x=O(\varepsilon)$, with $\varepsilon \ll 1$, for example $\varepsilon=O\left(R e^{-1}\right)$ in boundary layer flows. To make use of this fact, a single-frequency three-dimensional disturbance is expressed in the form (c.c. denotes complex conjugate)

$$
\tilde{\boldsymbol{q}}(x, y, z, t)=\hat{\boldsymbol{q}}(x, y, z) \exp [\mathrm{i} \theta(x, t)]+\text { c.c. },
$$

where $\hat{\boldsymbol{q}}(x, y, z)=(\hat{\rho}, \hat{u}, \hat{v}, \hat{w}, \hat{T})^{\mathrm{T}}$ is the vector comprising the amplitude functions of linear density, velocity components and temperature perturbations and with $\theta$ satisfying

$$
\theta(x, t)=\int_{x} \alpha\left(x^{\prime}\right) \mathrm{d} x^{\prime}-\omega t .
$$

Within the PSE-3D approximation, the streamwise derivatives of $\hat{\boldsymbol{q}}$ take the form

$$
\frac{\partial^{k} \tilde{\boldsymbol{q}}}{\partial x^{k}}=\mathrm{i}^{k}\left[\alpha^{k} \hat{\boldsymbol{q}}-\mathrm{i} k \alpha^{k-1} \frac{\partial \hat{\boldsymbol{q}}}{\partial x}-\mathrm{i} \frac{k(k-1)}{2} \alpha^{k-2} \frac{\mathrm{d} \alpha}{\mathrm{d} x} \hat{\boldsymbol{q}}\right] \exp [\mathrm{i} \theta(x, t)],
$$

and for the first and second derivative, the above expression becomes

$$
\frac{\partial \tilde{\boldsymbol{q}}}{\partial x}=\left(\mathrm{i} \alpha \hat{\boldsymbol{q}}+\frac{\partial \hat{\boldsymbol{q}}}{\partial x}\right) \exp [\mathrm{i} \theta(x, t)]
$$


and

$$
\frac{\partial^{2} \tilde{\boldsymbol{q}}}{\partial x^{2}}=\left(-\alpha^{2} \hat{\boldsymbol{q}}+2 \mathrm{i} \alpha \frac{\partial \hat{\boldsymbol{q}}}{\partial x}+\mathrm{i} \frac{\mathrm{d} \alpha}{\mathrm{d} x} \hat{\boldsymbol{q}}\right) \exp [\mathrm{i} \theta(x, t)] .
$$

Substituting (2.7) and the first and second streamwise derivatives (2.10)-(2.11) into the linearized Navier-Stokes equations, and neglecting terms of $O\left(\varepsilon^{2}\right)$ such as $\alpha_{x} \hat{\boldsymbol{q}}_{x}, \hat{\boldsymbol{q}}_{x x}$, $\alpha_{x x}$ and higher derivatives with respect to $x$ or streamwise derivatives in the viscous part of the equations, the compressible linear PSE-3D equations are written in compact form as

$$
\boldsymbol{L} \hat{\boldsymbol{q}}+\boldsymbol{M} \frac{\partial \hat{\boldsymbol{q}}}{\partial x}=0 .
$$

An ambiguity exists in the PSE formulation, in which the changes in amplitude along the slow spatial direction can be contained both in the amplitude function $\hat{\boldsymbol{q}}$ or in the phase function of the ansatz (2.8), $\theta$. A normalization condition is required in order to close the formulation of the problem; see Herbert $(1994,1997)$ for classical PSE analysis, in which one homogeneous direction is assumed, for example the spanwise direction in boundary layer problems. In this work, the following normalization condition is used:

$$
\int_{\Omega} \rho_{b}^{1 / 2} \hat{V}^{\dagger} \frac{\partial\left(\rho_{b}^{1 / 2} \hat{V}\right)}{\partial x} \mathrm{~d} \Omega=0
$$

where $\hat{V}=(\hat{u}, \hat{v}, \hat{w})^{\mathrm{T}}$ and $\Omega$ is a $y-z$ plane. To a first-order approximation this normalization imposes that the kinetic energy of the shape functions remains independent of $x$. Thus, the amplitude growth is absorbed into the phase function, much like Chang et al. (1991) and Bertolotti, Herbert \& Spalart (1992), but now applied to two-dimensional amplitude functions $\hat{\boldsymbol{q}}(y, z)$.

The parabolization of equations is not entirely consistent, due to the term $\hat{p}_{x}$, and numerical instabilities will prevent the recovery of converged solutions. This term, associated with the left-running characteristic (for subsonic flows only) allows upstream influence in (2.12). In this work, the upstream propagation is suppressed in an equivalent manner to the incompressible limit, in which Li \& Malik (1997) showed that when using an implicit scheme along with sufficiently large streamwise steps, the upstream propagating modes are not captured in the numerical integration, thus permitting the stable integration of downstream-moving instabilities.

\subsubsection{Spatial BiGlobal analysis}

The spatial BiGlobal analysis will be necessary to obtain the shape function, wavenumber and damping/growth-rate, required as initial conditions of the PSE3D marching integration. Furthermore, its results offer a first approximation of the unstable linear modes of the flow under the assumption of locally parallel base flow. In order to proceed, the base flow is assumed to be locally independent of one spatial coordinate $x$ (but depending on the other two spatial directions, $y$ and $z$, in a coupled inhomogeneous manner) and therefore the unsteady three-dimensional infinitesimal perturbations, being inhomogeneous in $y$ and $z$ and periodic in $x$, may be written as

$$
\tilde{\boldsymbol{q}}(x, y, z, t)=\hat{\boldsymbol{q}}(y, z) \exp [\mathrm{i}(\alpha x-\omega t)]+\text { c.c. },
$$

with $\hat{\boldsymbol{q}}$ representing the vector of two-dimensional complex amplitude functions.

In the present spatial framework, $\omega$ is taken to be a real frequency parameter, while the complex eigenvalue $\alpha$ and the associated eigenvectors $\hat{\boldsymbol{q}}$ are sought. The 


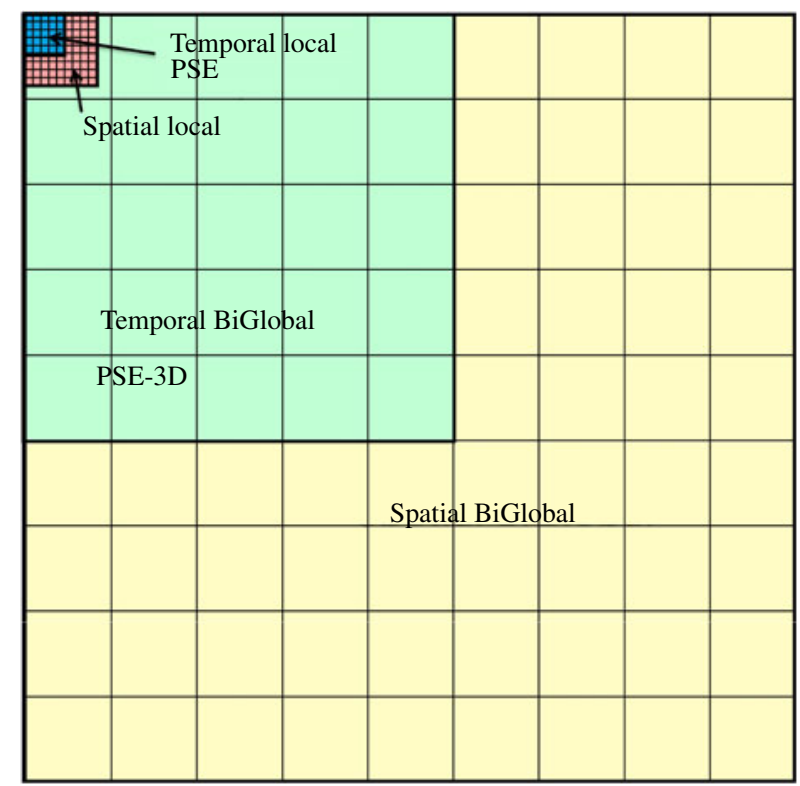

FIGURE 1. (Colour online) Schematic comparison of the leading dimensions of matrices discretizing local, classical PSE, BiGlobal and PSE-3D operators.

real part of the eigenvalue $\alpha_{r}$ is related to the wavenumber of the eigenmode along the homogeneous spatial direction $x, \alpha_{r}=2 \pi / L_{x}$, while the imaginary part is its growth/damping rate; a negative value of $\alpha_{i}$ indicates exponential growth of $\tilde{\boldsymbol{q}}$ in space, while $\alpha_{i}>0$ denotes decay of $\tilde{\boldsymbol{q}}$ in space.

The resulting two-dimensional partial derivative generalized eigenvalue problem (GEVP) is nonlinear on eigenvalue $\alpha$, but it is converted into a linear eigenvalue problem, which is larger in size by a factor equal to the degree of nonlinearity (see Theofilis 1995), using the companion matrix method (Bridges \& Morris 1984), in which an auxiliary vector is defined, $\hat{\boldsymbol{q}}^{+}=[\hat{\rho}, \hat{u}, \hat{v}, \hat{w}, \hat{T}, \alpha \hat{u}, \alpha \hat{v}, \alpha \hat{w}, \alpha \hat{T}]^{\mathrm{T}}$, and the resulting linear GEVP is

$$
\boldsymbol{A} \hat{\boldsymbol{q}}^{+}=\alpha \boldsymbol{B} \hat{\boldsymbol{q}}^{+} .
$$

The leading dimensions of the matrices discretizing the two-dimensional PDE of the PSE-3D (2.12) and spatial BiGlobal analysis GEVP (2.15) are (depending on the number of discretization nodes) several orders of magnitude larger than when solving the one-dimensional ODE of the classical PSE and local stability equations. Figure 1 shows a schematic comparison of their leading dimensions for compressible flow regimes.

\section{Numerical considerations}

\subsection{Details of the Navier-Stokes simulations}

The laminar-turbulent transition induced by a sharp-edged isolated roughness element in an $M=2.5$ boundary layer is analysed in two parts. For the first part of the investigation, which focuses on the linear instability of the wake induced by the isolated roughness element, the full Navier-Stokes equations are simulated and 


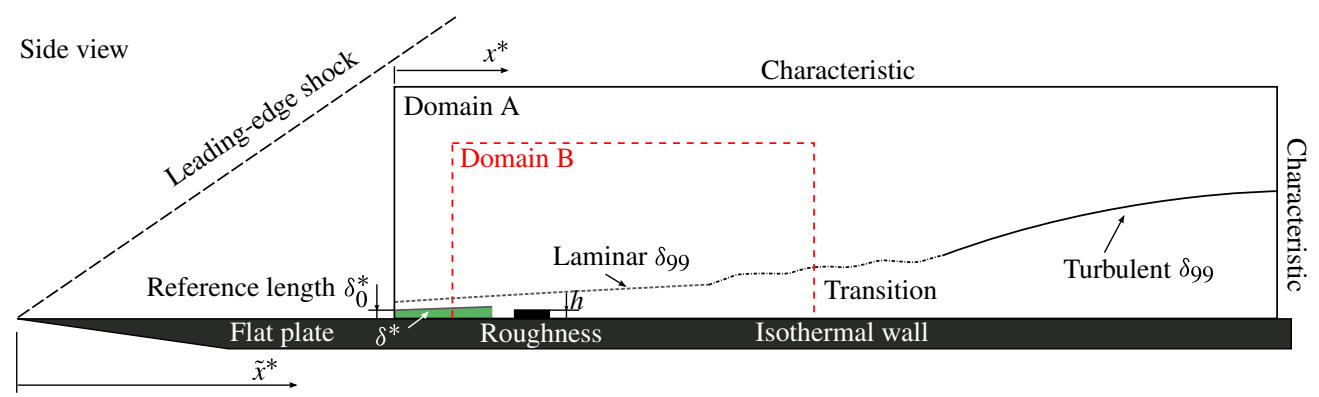

FIgure 2. (Colour online) Computational domain and boundary conditions. Domain A is used for simulations of the complete transition process while domain B is used for the study of the roughness wake instability.

compared with BiGlobal and PSE-3D simulations. The study includes the effects of two roughness elements with different non-dimensional heights, namely $h=0.5$ (case L0.5) and $h=1.0$ (case L1.0), and the case of a smooth flat plate ( $h=0$, case L0), which was considered for reference. The two roughness heights expressed in terms of $\delta_{99}$ of the surrounding undisturbed boundary layer at the streamwise location of the roughness element are $h^{*} / \delta_{99}=0.22$ and $h^{*} / \delta_{99}=0.44$ for cases L0.5 and L1.0 respectively. In all the cases the roughness elements have length $L=6.0$ and width $W=6.0$ and the Reynolds number of the simulation, based on the laminar displacement thickness at the inlet of the computational domain $\delta_{0}^{*}$ (more details in $\S 3.1 .1$ ), is $R e_{\delta_{0}^{*}}=3300$. This leads to roughness Reynolds numbers $R e_{h}=170$ and $R e_{h}=791$ for $h=0.5$ and $h=1.0$, respectively, and modified roughness Reynolds numbers (Bernardini et al. 2012) $R e_{h}^{*}=169$ and $R e_{h}^{*}=788$. In the second part of the study an additional DNS is carried out for the $h=1.0$ roughness element (case NL1.0), where the instability of the roughness wake is followed all the way to breakdown to turbulence. More details can be found in De Tullio \& Sandham (2012), which also includes results for a calculation at $M=6$.

\subsubsection{Computational domain and boundary conditions}

The geometry of the problem under consideration consists of a flat plate with a sharp-edged rectangular isolated roughness element. A two-dimensional sketch of the configuration is shown in figure 2. Two computational domains, denoted as domain A and domain $\mathrm{B}$, are used for the analysis of the different stages of the laminar-turbulent transition process. Domain A is used for the study of the nonlinear breakdown to turbulence, while domain B is employed to investigate the initial linear instability of the wake forming downstream of the roughness element. Both computational domains are placed downstream of the flat-plate leading edge and do not include the associated weak shock.

The roughness element is placed at a non-dimensional distance $x_{h}=x_{h}^{*} / \delta_{0}^{*}=50$ from the inflow of domain A. Here, $x_{h}^{*}$ is the dimensional streamwise position of the roughness leading edge in a reference frame positioned at the inflow of domain A and $\delta_{0}^{*}$ is the laminar displacement thickness at the same streamwise position. The laminar displacement thickness $\left(\delta^{*}\right)$ and the boundary layer thickness $\left(\delta_{99}\right)$ grow in the streamwise direction according to

$$
\frac{\delta^{*}\left(\tilde{x}^{*}\right)}{\delta_{0}^{*}}=\Delta \frac{\sqrt{2 R e_{\tilde{x}^{*}}}}{\operatorname{Re}_{\delta_{0}^{*}}} \quad \text { and } \quad \frac{\delta_{99}\left(\tilde{x}^{*}\right)}{\delta^{*}\left(\tilde{x}^{*}\right)}=\frac{\Delta_{99}}{\Delta},
$$


where

$$
\operatorname{Re}_{\tilde{x}^{*}}=\frac{1}{2}\left(\frac{\operatorname{Re}_{\delta_{0}^{*}}}{\Delta}\right)^{2}+\operatorname{Re}_{\delta_{0}^{*}} \frac{x^{*}}{\delta_{0}^{*}}
$$

Equations (3.1) and (3.2) were derived from the similarity solution (see the section on the Illingworth transformation in White 2005). Note that $\tilde{x}^{*}$ is the dimensional streamwise coordinate in a reference frame positioned at the flat-plate leading edge. The scaling factors $\Delta$ and $\Delta_{99}$ are calculated as

$$
\Delta=\int_{0}^{\infty}\left(\frac{\rho_{\infty}^{*}}{\rho^{*}}-\frac{u^{*}}{U_{\infty}^{*}}\right) \mathrm{d} \eta \text { and } \Delta_{99}=\int_{0}^{\eta_{99}} \frac{\rho_{\infty}^{*}}{\rho^{*}} \mathrm{~d} \eta
$$

where $\eta=\left[\left(\rho_{\infty}^{*} U_{\infty}^{*}\right) /\left(2 \mu_{\infty}^{*} \widetilde{x}^{*}\right)\right]^{1 / 2} \int_{0}^{y^{*}}\left(\rho^{*}\right) /\left(\rho_{\infty}^{*}\right) \mathrm{d} y^{*}$ is the non-dimensional similarity coordinate and $\eta_{99}$ is obtained at $y_{99}^{*}$, where $u^{*} / U_{\infty}^{*}=0.99$. Equation (3.2) provides a straightforward method of converting from the dimensionless simulation data to a reference system based on the flat plate that would be used in an experiment. For the cases considered in this work $\Delta=2.897$ and $\Delta_{99}=5.874$. All the lengths reported in the following are made non-dimensional using $\delta_{0}^{*}$, so that domain $\mathrm{B}$ is considered as a subset of domain A.

Domain A was designed to accommodate the turbulent wedge evolution in all directions and has dimensions $L_{x} \times L_{y} \times L_{z}=250 \times 20 \times 60$. In particular, the wall-normal domain size is about four times bigger than the turbulent boundary layer thickness at the outflow boundary. The dimensions of domain B are $L_{x} \times L_{y} \times L_{z}=100 \times 16 \times 20$. The streamwise extent was chosen to obtain a significant linear growth of the most unstable disturbances, while the wall-normal domain size was designed so that any residual reflections coming from the top boundary would hit the outflow boundary without affecting the stability characteristics of the flow. The spanwise domain size was chosen to include the most unstable smooth flat-plate boundary layer mode, which has a spanwise wavelength of $\lambda_{z} \approx 20$.

The spanwise domain boundaries are treated with periodic boundary conditions, while the walls are considered no-slip and isothermal with $T_{w}=T_{a d}=2.05$, where $T_{a d}$ is the laminar adiabatic-wall temperature. The reflection of waves from the domain external boundaries is minimized by using integrated and standard characteristic conditions for the top and outflow boundaries, respectively. The standard characteristic conditions used here are those originally derived by Thomson (1987, 1990), whereby the rates of change of incoming characteristics are set to zero at characteristic boundaries. The inflow is initialized with a compressible laminar similarity solution and a pressure extrapolation boundary condition is then applied, whereby in the subsonic region of the boundary layer the inflow conservative variables are calculated by extrapolating the pressure from within the domain using a linear approximation. The boundary condition at the inflow changes to a prescribed time-varying condition when introducing inflow disturbances. To this end, the time-converged inlet flow field obtained with the extrapolation boundary condition is used as the base flow over which disturbances are superimposed. The methodology used to introduce disturbances in the flow field is the subject of $\S 3.1 .3$.

\subsubsection{Grid generation}

All the numerical grids employed in this work were stretched in the wallnormal direction using the following relation between the computational uniform grid 


$\begin{array}{llcccccc}\text { Case } & N_{x} & N_{y}\left(n_{y}^{r}\right) & N_{z} & b_{y} & \Delta x([\min , \max ]) & \Delta z([\min , \max ]) \Delta x^{+}, \Delta y_{\min }^{+}, \Delta z^{+} \\ \text {L0 } & 501 & 191(-) & 100 & 3.40 & {[0.20,0.20]} & {[0.20,0.20]} & - \\ \text { L0.5 } & 659 & 191(26) & 241 & 3.38 & {[0.06,0.21]} & {[0.05,0.20]} & - \\ \text { L1.0 } & 659 & 205(46) & 241 & 3.20 & {[0.06,0.21]} & {[0.05,0.20]} & - \\ \text { NL1.0 } & 1989 & 222(61) & 547 & 3.94 & {[0.06,0.12]} & {[0.05,0.15]} & 6.3,0.7,2.6\end{array}$

TABLE 1. Computational grids. $N_{x}, N_{y}$ and $N_{z}$ are the number of points in the streamwise, wall-normal and spanwise directions, while $n_{y}^{r}$ is the number of points for $0 \leqslant y \leqslant h$. The length and width of the roughness elements are resolved using $n_{x}^{r}=101$ and $n_{z}^{r}=121$ points, respectively, in all cases. The values of $\Delta x^{+}, \Delta y_{\min }^{+}$and $\Delta z^{+}$for case NL1.0 are calculated in a region of turbulent flow at the roughness mid-plane.

$(0<\eta<1)$ and the physical non-uniform grid $\left(0<y<L_{y}\right)$ :

$$
y=L_{y} \frac{\sinh \left(b_{y} \eta\right)}{\sinh b_{y}},
$$

where $b_{y}$ is the stretching factor. In the cases including a roughness element the value of $b_{y}$ was iteratively determined (close to a target) by imposing an integer number of grid points $\left(n_{y}^{r}\right)$ below the roughness and $y\left(n_{y}^{r}\right)=h$, where $h$ is the non-dimensional height of the roughness.

The distribution of grid points in the streamwise and spanwise directions is uniform in the cases involving clean flat plates, whereas for the roughness cases it was designed to adequately resolve the laminar flow near the roughness element and the turbulent flow downstream of it. The level of grid refinement necessary near the roughness, both for stability reasons and because of the high gradients induced by its sharp edges, requires the use of grid stretching in all directions in order to maintain a reasonable computational effort. Therefore, in the horizontal directions the computational grid presents regions of constant grid spacing with different levels of grid refinement, which are linked together by polynomials of the ninth degree. The objective is to link two uniform grids with different grid spacings using a polynomial function obtained by imposing $C^{4}$ continuity of the resulting stretching function. It should also be noted that, while requiring extensive grid refinement, a sharp roughness element facilitates the generation of high-quality numerical grids.

Table 1 provides details of the numerical grids employed in each case, while a closeup view of the numerical grid surrounding the roughness element is given in figure 3 , giving an indication of the grid-stretching in all directions. The grids employed for the instability study (cases L0, L05 and L1.0) were chosen after a grid-convergence study performed by comparing the results obtained using the reference grid (given in table 1) and a coarse grid with half the number of points in each direction. The effects of grid refinement on the base flow and its stability characteristics for case L1.0 are shown in figures $4(a)$ and $4(b)$, respectively. It can be seen that the differences between the reference and the coarse grids are negligible everywhere except for the disturbance growth near the computational domain outflow. As will be shown later, the results obtained in this region are not relevant for the study of the linear instability of the flow as the disturbance evolution becomes affected by nonlinear interactions. For case NL1.0 we note that the worst case values of $\Delta x^{+}, \Delta y_{\min }^{+}$and $\Delta z^{+}$, given in the last column of table 1, are better than most fully resolved DNS studies (see for example Pirozzoli, Grasso \& Gatski 2004; Muppidi \& Mahesh 2012). 


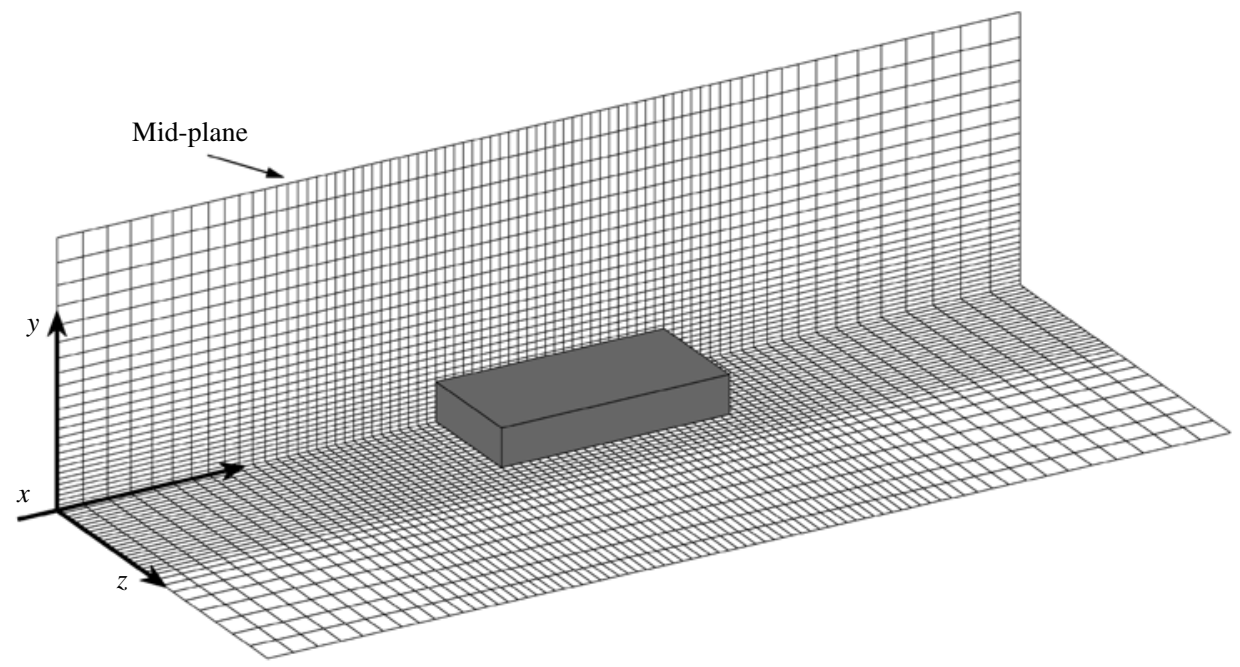

FIGURE 3. Detail of the numerical grid employed near the roughness element. Only every four grid points in the streamwise and spanwise directions and every six in the wall-normal direction are shown.
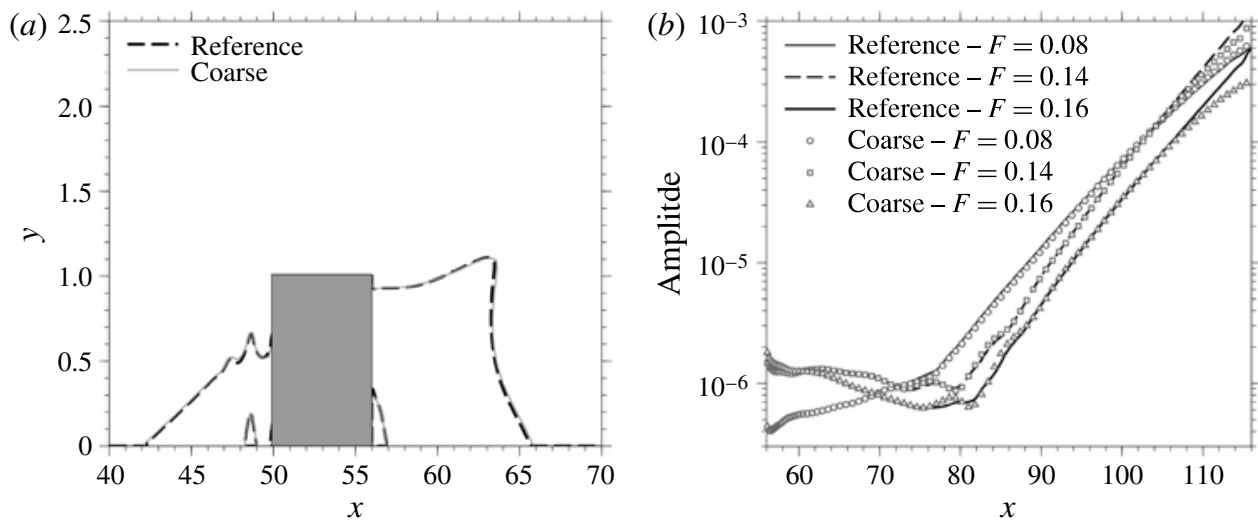

FIGURE 4. Grid convergence study. ( $a$ ) Contours of $u_{b}=0$ showing the roughness-induced separation bubbles at the roughness centreline (note that the $y$-axis has been stretched for clarity). (b) Amplitude of the Fourier-transformed (in time) wall-pressure, integrated across the span, for a selection of unstable frequencies.

\subsubsection{Disturbance generation}

During the linear instability study the laminar boundary layer base flow is subjected to a time-varying density disturbance imposed at the inflow boundary of domain B, which is placed at $x_{0}=16$, so that the inflow condition for density reads

$$
\rho\left(x_{0}, y, z, t\right)=\rho_{b}\left(x_{0}, y, z\right)+a G_{w} \sum_{m=1}^{M} \sum_{n=1}^{N} \cos \left(\beta_{m} z+\phi_{m}\right) \cos \left(\omega_{n} t+\psi_{n}\right),
$$

with $N=16$ and $M=6$ as the number of frequencies and spanwise wavenumbers respectively, $\beta_{m}=m 2 \pi / L z, \omega_{n}=n 2 \pi F_{0}$ with $F_{0}=0.02$. Note that the non-dimensional 
frequency $F=f^{*} \delta_{0}^{*} / U_{\infty}^{*}$, where $f^{*}$ is the dimensional frequency (cycles per second), takes the form of a Strouhal number. The phases $\phi_{m}$ and $\psi_{n}$ are set to random numbers between 0 and $2 \pi$ in order to avoid local peaks in the disturbance signal which might trigger undesired nonlinearities. The damping function $G_{w}=1-\exp \left(-y^{3}\right)$ was used to control the disturbance location with respect to the boundary layer; it is greater than zero inside the boundary layer and zero at the wall. An additional damping was employed to drive the forcing function to zero at the top domain boundary to avoid the onset of numerical oscillations. A small amplitude of $a=2 \times 10^{-6}$ is used to ensure the linearity of the dominating transition mechanisms, at least for the initial stages of the instability.

For the analysis of the nonlinear breakdown to turbulence downstream of the roughness element (in §5) an alternative acoustic broadband disturbance was placed in the free stream, upstream of the roughness element, by adding the following forcing term to the continuity equation;

$$
\sigma_{f}(x, y, z, t)=a \exp \left(-r^{2}\right) \sum_{m=1}^{M} \sum_{n=1}^{N} \cos \left(\beta_{m} z+\phi_{m}\right) \sin \left(\omega_{n} t+\phi_{n}\right) .
$$

The coordinate $\tilde{r}$ is defined as $r^{2}=\left[\left(x-x_{f}\right)^{2}+\left(y-y_{f}\right)^{2}\right] / L_{f}$, where $L_{f}=1.0$ determines the radius of the forcing region and $x_{f}=25.0$ and $y_{f}=10.0$ its centre. In this case the disturbance signal is composed of $N=18$ frequencies with $F_{0}=0.06$ and $M=25$ spanwise wavenumbers. The amplitude of the forcing function was increased to $a=6 \times 10^{-5}$ in this case in order to move the point of breakdown to turbulence upstream, while still retaining a clear linear stage of disturbance growth. The amplitude of the forced disturbances can more easily be increased without introducing numerical noise when using (3.6).

\subsubsection{The compressible Navier-Stokes solver}

The compressible Navier-Stokes equations (2.3) are solved numerically using highorder finite differences. The spatial discretization is treated using a standard fourthorder central difference scheme to calculate derivatives at internal points, while close to boundaries a stable boundary treatment by Carpenter, Nordstrom \& Gottlieb (1999) is applied, giving overall fourth-order accuracy. Time integration is based on a thirdorder compact Runge-Kutta method (Wray 1990) and an entropy splitting approach developed by Sandham, Li \& Yee (2002) is used to split the inviscid flux derivatives into conservative and non-conservative parts, thereby improving numerical stability. The code has multi-block capabilities and is made parallel (both intra- and inter-block) using the message passing interface (MPI) library. Details on the implementation of the numerical schemes can be found in Li (2003) and Jones (2008). The multi-block version of the code used in this work has been extensively validated (see for example Yao et al. 2009; De Tullio \& Sandham 2010).

\subsection{Numerical treatment of the instability analyses}

\subsubsection{Solution procedure of PSE-3D}

The parabolic PDE system of (2.12) is solved by marching along the streamwise direction. The derivative in the marching direction is approximated by the implicit backward Euler scheme

$$
\left(\hat{\boldsymbol{q}}_{x}\right)_{j+1} \approx \frac{1}{\Delta x_{j}}\left(\hat{\boldsymbol{q}}_{j+1}-\hat{\boldsymbol{q}}_{j}\right)
$$


where $j \geqslant 0$ is the step index and $\hat{\boldsymbol{q}}_{j}=\hat{\boldsymbol{q}}_{j}(y, z)=\hat{\boldsymbol{q}}\left(x_{j}, y, z\right)$. Equation (2.12) becomes

$$
\left[\Delta x_{j} \boldsymbol{L}_{j+1}^{n}+\boldsymbol{M}_{j+1}^{n}\right] \hat{\boldsymbol{q}}_{j+1}^{n}=\boldsymbol{M}_{j+1}^{n} \hat{\boldsymbol{q}}_{j},
$$

where $n$ is the iteration index. Starting with an initial guess $\alpha_{j+1}^{0}=\alpha_{j}$, according to the first approximation $\alpha_{x}=0, \hat{\boldsymbol{q}}_{j+1}^{0}$ is obtained from (2.12). Then, the normalization condition (2.13) is used to estimate a new $\alpha_{j+1}$ :

$$
\alpha_{j+1}^{n+1}=\alpha_{j+1}^{n}-\frac{i}{\Delta x_{j}} \frac{\int_{\Omega}\left(\rho_{b}\right)_{j+1}^{1 / 2} \hat{V}_{j+1}^{\dagger n}\left[\left(\rho_{b}\right)_{j+1}^{1 / 2} \hat{V}_{j+1}^{n}-\left(\rho_{b}\right)_{j}^{1 / 2} \hat{V}_{j}\right] \mathrm{d} \Omega}{\int_{\Omega}\left(\rho_{b}\right)_{j+1}\left|\hat{V}_{j+1}^{n}\right|^{2} \mathrm{~d} \Omega} .
$$

Once the wavenumber is updated, (3.8) is solved for the new shape functions, and the value of $\alpha$ is recalculated. The iteration continues until the normalization condition is satisfied to within some specified residual $r$ (i.e. $\left|\alpha^{n+1}-\alpha^{n}\right|<r=10^{-8}$ in the present computations).

\subsubsection{Eigenvalue computation of spatial BiGlobal problem}

The elliptic spatial BiGlobal problem, written as a GEVP (2.15), is solved using the Arnoldi algorithm (Saad 1980), delivering a number of eigenvalues on the vicinity of a specific estimate value, usually around the unstable/least-stable eigenvalue. The computational cost is significantly reduced by employing the Arnoldi algorithm instead of computing the whole eigenspectrum by the classical QZ method (Wilkinson 1965). More details can be found in the literature (Saad 1980; Theofilis 2003).

\subsubsection{Large matrix inversion and spatial discretization}

The inversion of the matrices discretizing the two-dimensional PDE of the PSE3D equations (2.12) and the spatial BiGlobal analysis GEVP (2.15), with leading dimension $O\left(10^{4}-10^{5}\right)$, is performed using the parallelizable sparse matrix linear algebra package MUMPS (Amestoy et al. 2001, 2006) and the SPARSKIT2 library (Saad 1994). These libraries exploit the high level of sparsity pattern offered by the finite difference spatial differentiation, improving substantially on numerical efficiency while keeping accuracy; see the work of Paredes et al. (2013) for more details.

The two directions of the plane normal to the streamwise marching direction $(y, z)$ are discretized using high-order finite difference methods. For the spanwise directions two different schemes are used. When considering the whole spanwise extent of the computational domain, periodic boundary conditions are imposed and standard finite difference schemes are used for the spatial discretization. On the other hand, when only half the spanwise extent of the computational domain is discretized, high-order finite difference numerical schemes of order $q$ (FD-q) for non-symmetric domains, recently developed by Hermanns \& Hernández (2008), are used in order to study the behaviour of symmetric and anti-symmetric modes (with respect to the roughness mid-plane $z=10$ ) separately. This approach also leads to a reduction of the computational cost of the simulations. The wall-normal direction is discretized using the FD-q method. These methods are used because spectral-like accuracy is recovered, outperforming spectral collocation methods for stability analysis calculations in terms of accuracy and computational efficiency (Paredes et al. 2013). The eighth- and tenthorder schemes, FD-q8 and FD-q10, were found to provide an optimal compromise between accuracy and efficiency (Paredes et al. 2011).

Appropriate mappings between the finite difference grids $(\xi \in[-1,1])$ and the physical domain is needed. Since the boundary layer problem requires clustering of 
points at the wall, the equation used to map the calculation domain $y \in\left[0, y_{\infty}\right]$ into the FD-q grid is

$$
y_{j}=l \frac{1-\xi_{j}}{1+s+\xi_{j}}, \quad s=2 l / y_{\infty}, \quad l=\frac{y_{\infty} y_{h}}{y_{\infty}-2 y_{h}},
$$

where $y_{\infty}$ is the location where the calculation domain is truncated and $y_{h}$ is the domain location that splits the number of discretization points into two halves. In this direction, the selected mapping parameters are $y_{h}=2.5, y_{\infty}=16$. For the spanwise direction, when the whole spanwise extent of the computational domain is considered, the clustering of points is required at $z_{0}=10$ and another transformation is used in order to map the calculation domain grid $z \in\left[z_{0}-z_{m}, z_{0}+z_{m}\right]$ into the grid $\xi \in[-1,1]$ :

$$
z=z_{0}+z_{m} \frac{\tan \left(\frac{c_{z} \pi}{2} \xi\right)}{\tan \left(\frac{c_{z} \pi}{2}\right)},
$$

where $c_{z}$ is the stretching factor, which is set to $c_{z}=0.75$. When considering only half of the spanwise extent of the computational domain, the axis origin is translated to the mid-plane and the transformation of (3.10) is used with parameters $z_{\infty}=10$ and $z_{h}=2$.

\section{Discussion of the results}

\subsection{The laminar base flow}

The steady-state laminar base flow over the roughness element is analysed here by comparison with the flow over a clean flat plate. Our interest is to highlight the modifications induced by the roughness element, which will determine the stability characteristics of the laminar flow.

The roughness element induces two regions of three-dimensional separated flow, located immediately upstream and downstream of it, as can be seen in figures $5(a)$ and 5(b), for cases L1.0 and L0.5 respectively, through isosurfaces of small negative streamwise velocity in blue (online). The wake of the roughness element is characterized by the presence of regions of high streamwise vorticity. A pair of streamwise vortices are generated at the roughness edges due to a small difference in pressure between the top (higher) and the sides (lower) of the roughness, clearly seen in figure $5(b)$ for case L0.5 (edge vortices). For case L1.0, in addition to the edge vortices, a pair of strong counter-rotating streamwise vortices form as the flow reattaches downstream of the roughness, visible in figure $5(b)$ near the roughness mid-plane. Figure 6 shows shaded contours of $u_{s}=\left[\left(\partial u_{b} / \partial y\right)^{2}+\left(\partial u_{b} / \partial z\right)^{2}\right]^{1 / 2}$ superimposed by contour lines of $u_{b}=0.38$ (orange line), at three different streamwise positions and for the two roughness heights considered (subscript $b$ refers to the base flow as defined in $\$ 2.2$ ). For case L1.0 the strong streamwise vortices induced downstream of the roughness element lift up low-momentum fluid from the near-wall region and give rise to a low-velocity streak away from the wall, near the mid-plane of the roughness element, as shown in figures $6(a), 6(c)$ and $6(e)$. As the low-velocity streak forms, it induces a three-dimensional detached high-shear layer in its surroundings, leading to a highly unstable roughness wake, as will be shown in the following sections. Note that the shape of the low-velocity streak changes notably along the streamwise extent of the computational domain, suggesting that non-parallel flow effects might play 


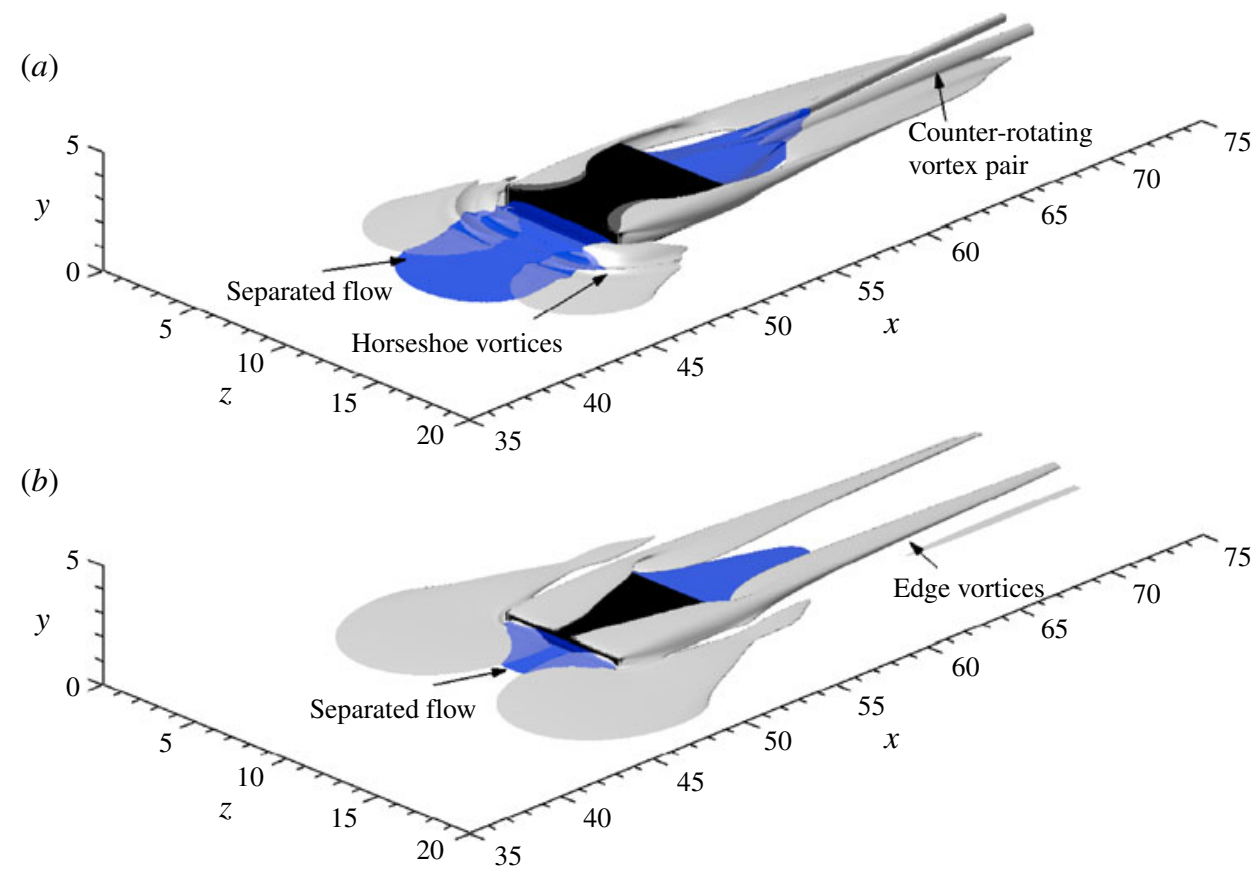

FIGURE 5. (Colour online) Basic flow topology around the roughness element: $(a)$ cases L1.0 and NL1.0, $(b)$ case L0.5. Isosurfaces of small negative $u_{b}$ show the separated flow regions in mid grey (blue online). Grey isosurfaces of $\omega_{x}= \pm 0.28$ for $(a)$ and $\omega_{x}= \pm 0.08$ for $(b)$, positive on the right and negative on the left part of the computational domain looking downstream, show the roughness-induced vortices.

(a) 3

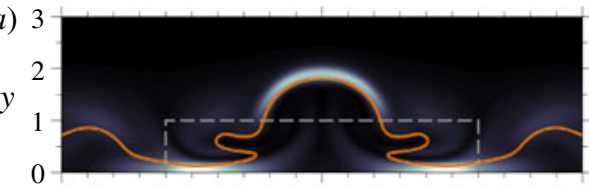

(c)

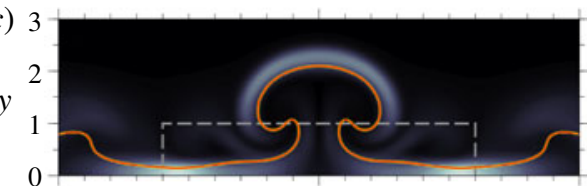

(e)

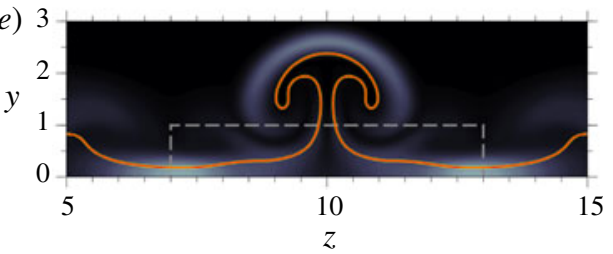

(b)

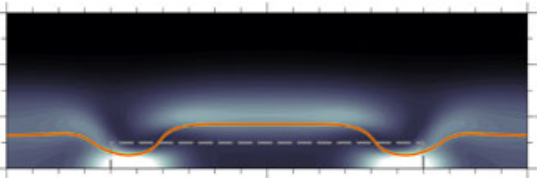

(d)

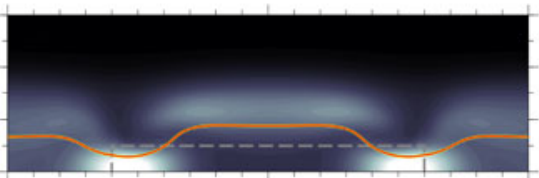

$(f)$

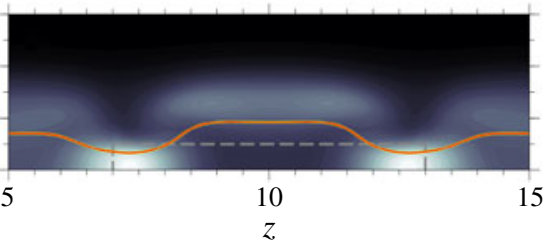

FIgURE 6. (Colour online) Contours of $u_{s}$ in cross-flow planes at $(a, b) x=76.025$, $(c, d) x=93.665$ and $(e, f) x=114.665$, showing the localized shear generated by the roughness-induced counter-rotating vortices. The orange lines show contours of $u_{b}=0.38$. $(a, c, e)$ Case L1.0, and $(b, d, f)$ case L0.5. 

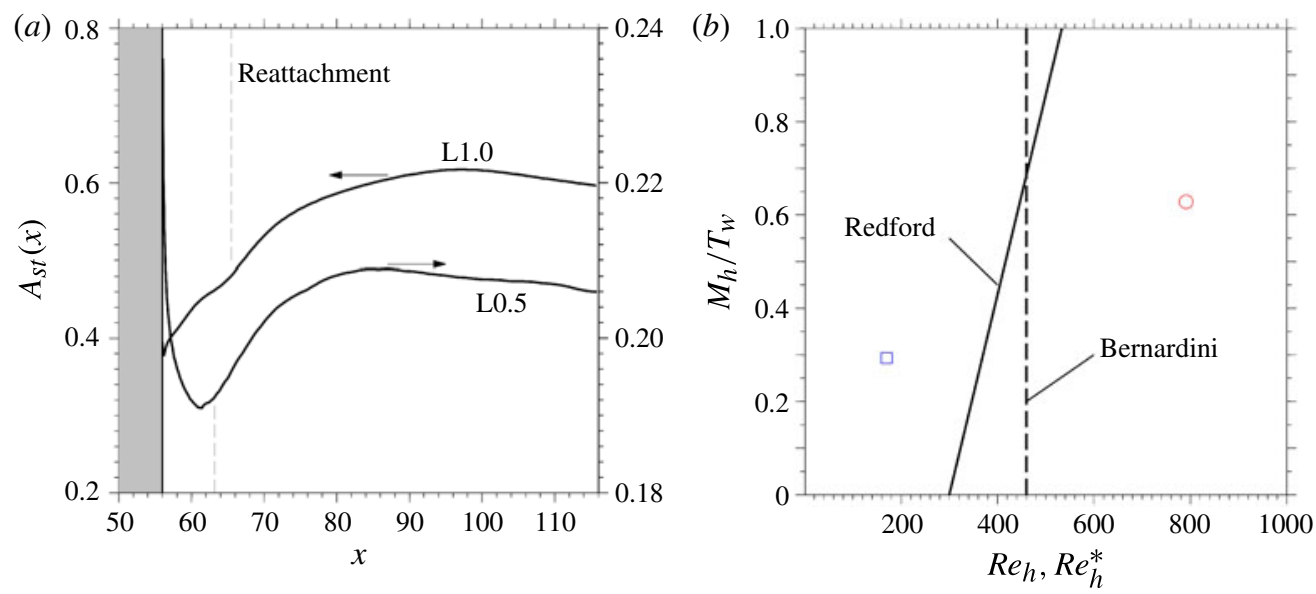

FIGURE 7. Predicting roughness effects on transition using $(a)$ the streak amplitude method of Andersson et al. (2001) and (b) the transition maps of Redford et al. (2010) and Bernardini et al. (2012).

an important role in the determination of the stability characteristics of the wake. Figures $6(b), 6(d)$ and $6(f)$ show the steady laminar base flow downstream of a roughness element with $h=0.5$ (case L0.5). It can be seen that, in comparison with case L1.0, the effects of the edge vortices on the boundary layer flow are much weaker, and once the roughness wake is formed its shape appears to be independent of the $x$-position.

The strength of the low-velocity streak in the two cases analysed is calculated using the definition for the streak amplitude

$$
A_{s t}(x)=\frac{1}{2}\left[\max _{y, z}\left(u_{b}(x, y, z)-u_{b}\left(x, y, z_{0}\right)\right)-\min _{y, z}\left(u_{b}(x, y, z)-u_{b}\left(x, y, z_{0}\right)\right)\right],
$$

first proposed by Andersson et al. (2001), where $z_{0}=0$ and hence at each $x$-position the wake flow is compared with the surrounding boundary layer flow. The results for the two roughness heights are provided in figure $7(a)$. In both cases the streak amplitude undergoes a sudden growth immediately downstream of the roughnessinduced separation bubble, reaching a maximum and decaying further downstream, in a typical transient growth by 'lift-up' behaviour. The maximum streak amplitude for case L0.5 is approximately $21 \%$ of the free-stream velocity and is obtained at $x \approx 84$. According to the findings of Andersson et al. (2001) (based on incompressible flow), both sinuous and varicose modes are stable in this case. For case L1.0, on the other hand, the maximum streak amplitude reaches $\sim 62 \%$ at $x \approx 97$, well above the streak stability limits of 26 and $37 \%$ identified by Andersson et al. (2001) for the growth of sinuous and varicose modes, respectively. It is worth noting that, as already pointed out by Groskopf et al. (2009), the definition of $A_{s t}$ in (4.1) does not resolve a streak from a vortex, hence in our case $A_{s t}$ initially (i.e. near the roughness trailing edge) contains contributions from both the streak and the counter-rotating vortex pair.

Figure 7(b) shows the two flow configurations analysed in relation to the transition criteria proposed by Redford et al. (2010) and Bernardini et al. (2012). The transitionmap plane is divided into two regions, one where roughness does not have an effect on transition (left) and one where transition is dominated by roughness effects (right), by the two critical lines (solid and dashed black lines in the figure). Both criteria predict 
negligible roughness effects for case L0.5 and roughness-induced laminar-turbulent transition for case L1.0.

\subsection{Linear instability of the roughness wake}

The instability of the wake developing downstream of the roughness element is analysed through NS, spatial BiGlobal and PSE-3D calculations. The NS results are analysed by expanding the disturbance field into frequencies $(F)$ and spanwise wavenumbers $\left(k_{z}\right)$. Fourier decomposition is carried out according to the discrete Fourier transform (DFT) formula

$$
\begin{gathered}
\breve{S}_{\eta, \xi}(x, y)=\frac{2}{L J} \sum_{j=0}^{J-1} \sum_{l=0}^{L-1} S\left(x, y, z_{l}, t_{j}\right) \exp \left[-2 \pi \mathrm{i} \frac{\eta}{J} j\right] \exp \left[-2 \pi \mathrm{i} \frac{\xi}{L} l\right] \\
\eta=0,1, \ldots, J-1 \text { and } \xi=0,1, \ldots, L-1
\end{gathered}
$$

where $J$ and $L$ are the total time and space samples and $S$ can be any flow variable. The indices $\eta$ and $\xi$ represent the discretized frequencies and spanwise wavenumbers, respectively. Non-dimensional frequencies can then be obtained as $F=\eta / \tau$, with $\eta=0,1, \ldots, J / 2-1$, where $\tau=50$ is one sampling period, corresponding to one forcing cycle. After reordering to place the zero-wavenumber component at the centre of the spanwise wavenumber spectrum, we obtain $k_{z}=2 \pi \xi / L_{z}$, with $\xi=-(L / 2-1), \ldots,-1,0,1, \ldots, L / 2-1$, where $L_{z}=20$ is the spanwise length of the computational domain. The normalization factor $2 /(L J)$ gives unit amplitude Fourier modes for a disturbance signal given by a sum of temporal and spatial sinusoidal waves. Recall that, here, the non-dimensional frequency $F$ is obtained from the dimensional frequency $f^{*}$ as $F=f^{*} \delta_{0}^{*} / U_{\infty}^{*}$. The standard frequency definition $F_{s}=2 \pi f^{*} \mu_{\infty}^{*} /\left(\rho_{\infty}^{*} U_{\infty}^{* 2}\right)$ used in linear stability studies can be recovered from $F_{s}=2 \pi F / \operatorname{Re}_{\delta_{0}^{*}}$.

In addition to the amplitude of the single Fourier modes $\left(|\breve{S}|_{\eta, \xi}(x, y)\right)$, the amplitude associated with each frequency is defined here as

$$
|\breve{S}|_{\eta,(\cdot)}(x, y)=\sum_{\xi=1}^{M}|\breve{S}|_{\eta, \xi}(x, y),
$$

and the boundary layer response to the inflow disturbances is quantified using Mack's disturbance energy norm (Mack 1969), which can be expressed in dimensionless form as

$$
\begin{aligned}
\mathscr{E}_{\eta}(x)= & \int_{0}^{\delta_{99}}|\breve{\rho}|_{0,(\cdot)}\left[|\breve{u}|_{\eta,(\cdot)}^{2}+|\breve{v}|_{\eta,(\cdot)}^{2}+|\breve{w}|_{\eta,(\cdot)}^{2}\right] \\
& +\frac{|\breve{\rho}|_{\eta,(\cdot)}^{2}|\breve{T}|_{0,(\cdot)}}{\gamma|\breve{\rho}|_{0,(\cdot)} M^{2}}+\frac{|\breve{T}|_{\eta,(\cdot)}^{2}|\breve{\rho}|_{0,(\cdot)}}{\gamma(\gamma-1)|\breve{T}|_{0,(\cdot)} M^{2}} \mathrm{~d} y .
\end{aligned}
$$

Note that the $\eta=0$ Fourier coefficients are associated with the laminar base flow. For the remainder of this paper the breve symbol (as in $\breve{S}$ ) will be used for quantities that are Fourier-transformed both in time and in the spanwise direction, while the hat symbol will be used for Fourier transforms over time only and for the linear eigenfunctions obtained from the stability analysis.

Figure 8 shows the base flow response to forcing frequencies $F=0.02, F=0.1$ and $F=0.24$ (chosen to represent the behaviour at the low, medium and high frequency 


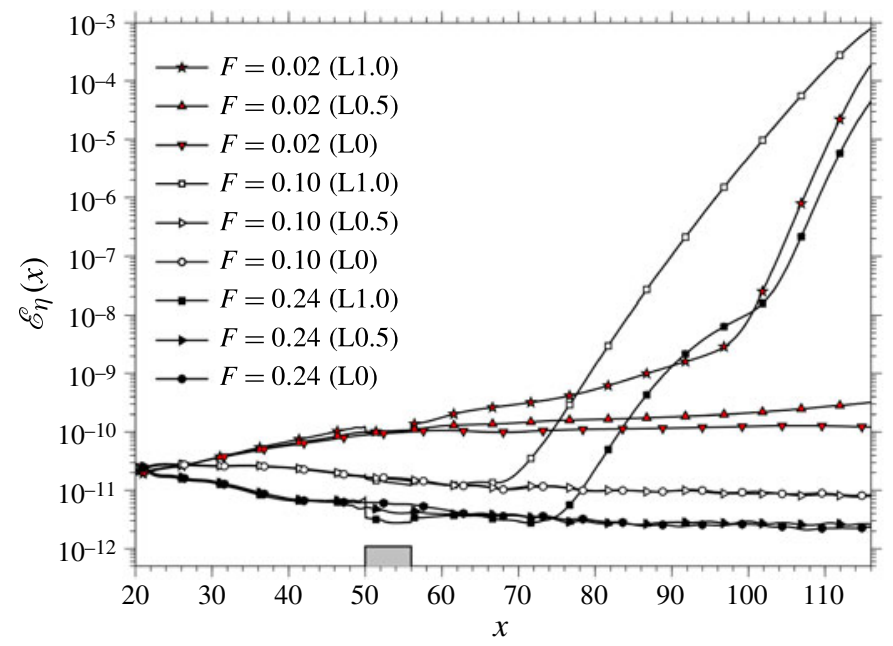

FIGURE 8. (Colour online) Streamwise evolution of disturbance energy for different roughness heights. The grey rectangle indicates the position of the roughness element.

ranges) for cases L0, L0.5 and L1.0. After the initial boundary layer receptivity, occurring immediately downstream of the inflow boundary, a secondary receptivity process takes place downstream of the roughness element as the mean flow changes abruptly, leading to a redistribution of disturbance energy into modes of the new base flow. This can be seen as a sudden amplification at about $x=60$, noticeable for $F=0.02$ in figure 8 , which is more pronounced for the low frequencies and for the taller roughness element. Both this and the subsequent linear growth-rate increase with roughness height. As the frequency increases the effect of the small roughness weakens until it becomes negligible. In fact, the $F=0.10$ and $F=0.24$ disturbances shown in figure 8 experience the same amplitude decay for cases L0.5 and L0, and the same is found to be true for all $F>0.04$. The effect of the small roughness element on the boundary layer stability is weak and limited to the low frequencies $(F=0.02$ and $F=0.04$ in our case). The low frequency response for case L0.5 departs slightly from that of case LO and is characterized by the excitation of neutral or slightly unstable disturbances (not shown) in the regions of relatively high spanwise shear induced near the sides of the roughness element (see for example figure $6 b$ near $z=6.5$ and $z=13.5$ ). These results suggest that transition to turbulence will not occur due to roughness effects in this case, as predicted by the transition maps of Redford et al. (2010) and Bernardini et al. (2012). In addition, the stability predicted by the streak amplitude criterion of Andersson et al. (2001) is confirmed for a compressible flow.

The roughness wake response to the incoming disturbances is drastically different for case L1.0. Disturbances grow quickly downstream of the roughness element, showing a maximum disturbance energy growth of more than seven orders of magnitude between the inflow and outflow boundaries, so that some of the most unstable modes have already reached finite amplitudes before the end of the computational domain. This leads to the increased growth-rate of $F=0.02$ and $F=0.24$ modes for $x>100$, which is due to nonlinear interactions of $F=0.10$, $F=0.12$ and $F=0.14$ disturbances. 

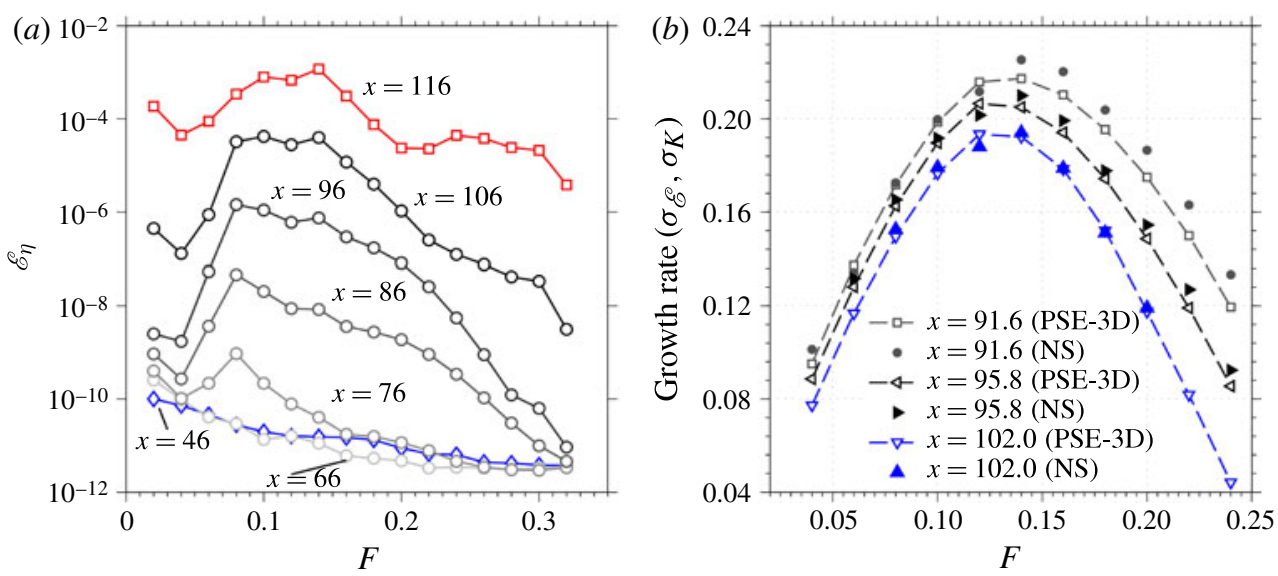

FIGURE 9. (Colour online) Disturbance energy growth inside the boundary layer as a function of frequency for case L1.0. (a) Disturbance energy at different streamwise positions for all forcing frequencies. (b) Disturbance growth-rate at different streamwise positions for a selection of the most unstable frequencies ( $\sigma_{\mathscr{E}}$ for NS and $\sigma_{K}$ for PSE-3D).

The roughness wake is receptive to a broad frequency band, as can be seen in figure $9(a)$, which shows the disturbance amplitude associated with the different forcing frequencies at different streamwise locations. At $x=46$ (blue line (online)) the disturbance amplitudes inside the boundary layer are high at $F=0.02$, corresponding to the only unstable boundary layer mode excited, and decrease with frequency. Immediately downstream of the roughness element $(x=66)$, frequencies $F=0.02$ and $F=0.04$ experience a sudden amplification while the high frequencies are either damped by the roughness or keep decreasing due to their inherent stability. A peak centred at $F=0.08$ appears near $x=76$, indicating that the roughness wake mode with $F=0.08$ has the highest receptivity. However, further downstream higher frequencies start to grow quickly, and by $x=116$ disturbances in the range $0.08 \leqslant F \leqslant 0.16$ are the most amplified. The high amplitudes shown in figure $9(a)$ at the low and high end of the frequency spectrum are again a consequence of nonlinear interactions between the most amplified linear modes.

Figure $9(b)$ compares the growth-rates of the dominant modes extracted from the PSE-3D results at different streamwise locations with the NS results for a selection of frequencies. The NS growth-rates are measured as

$$
\sigma_{\mathscr{E}}=\frac{1 \mathrm{~d} \ln \left[\mathscr{E}_{\eta}(x)\right]}{\mathrm{d} x} \text { for } \eta=1,2, \ldots, J / 2-1,
$$

while for the PSE-3D results the effective growth-rate, defined as

$$
\sigma_{K}=-\alpha_{i}+\frac{1}{2} \frac{\mathrm{d} \ln [K(x)]}{\mathrm{d} x}, \quad \text { with } K(x)=\int_{\Omega} \rho_{b}\left(\hat{u} \hat{u}^{\dagger}+\hat{v} \hat{v}^{\dagger}+\hat{w} \hat{w}^{\dagger}\right) \mathrm{d} \Omega,
$$

is used to account for the residual slow variation of disturbance kinetic energy $(K)$ with $x$. Note that the PSE-3D growth-rates can also be computed from the streamwise variation of the mode amplitudes, similarly to what was done for the NS results. The growth-rates obtained using the two methodologies were found to be practically indistinguishable from each other. This aspect was also reported by Bertolotti \& Herbert (1991), who showed that the use of normalization condition 
(2.13), and consequent definition of the effective growth-rate $\left(\sigma_{K}\right)$ does not change the results. Therefore the definition in (4.6) was used here for comparison with the NS results. Both NS and PSE-3D results show that disturbances with $F=0.14$ are the most unstable, with an averaged growth-rate of $\sigma_{K} \approx 0.21$ for $80 \leqslant x \leqslant 116$. This is roughly thirty times higher than the smooth flat-plate primary instability for the same Mach and Reynolds numbers, which has $F \approx 0.013$ and $-\alpha_{i} \approx 0.0075$ according to parallel linear stability theory. While the NS and PSE-3D growth-rates agree closely for $x \geqslant 95.8$, a slight disagreement is evident at $x=91.6$ for $F \geqslant 0.14$. This is believed to be due to the fact that, as will be shown in the following, multiple modes with different growth-rates contribute to the growth of $\mathscr{E}_{\eta}$ in the NS simulations. In the linear limit the disturbed flow field is given by the sum of these modes and $\sqrt{\mathscr{E}_{\eta}}$ can potentially grow faster than the most unstable mode, as is the case for $F \geqslant 0.14$ in figure $9(b)$. The mechanism is similar to that responsible for the transient growth of disturbances in the presence of non-orthogonal stable modes, only here two (or potentially more) unstable modes combine linearly and lead to a total disturbance energy growing faster than the most unstable mode during a certain transient.

The NS results clearly show that at least two different modes experience rapid amplitude growth during the linear instability of the wake of the roughness element. The three-dimensional structure of these modes is shown in figures $10(a)$ and $10(b)$, for $F=0.14$ and $F=0.24$ respectively, through isosurfaces of the real part of the Fourier-transformed $v$-velocity. It can be seen that the dominant mode at $F=0.14$ is characterized by a symmetric $v^{\prime}$ disturbance field, consistent with a varicose deformation of the low-velocity streak. In contrast, the disturbance field at $F=0.24$ shows an antisymmetric $v^{\prime}$, suggesting that an additional sinuous mode grows on the edge of the streak. It is therefore clear that the roughness wake sustains the growth of both sinuous and varicose instability modes, as predicted by the streak amplitude criterion. Note that a varicose mode appears to take over near the computational domain outflow in figure $10(b)$ for $F=0.24$. This is due to the fact that, as already shown in figure 8 , the growth of $F=0.24$ disturbances near the end of the computational domain is dominated by nonlinear interactions of the most amplified varicose modes.

A more complete picture of the modes taking part in the instability of the wake can be obtained by analysing the linear stability of $y-z$ slices of the roughness wake. Figure 11(a) gives the BiGlobal eigenspectrum obtained for $F=0.08$ at $x=93.66$. It can be seen that, in addition to the dominant varicose and sinuous modes, the structure of which is shown respectively in figures $11(b)$ and $11(c)$, the wake of the roughness element sustains the growth of four additional modes for the frequency and streamwise position considered. The modes shown in figures $11(d)$ and 11(e) belong to the family of modes growing in the three-dimensional high-shear layer surrounding the low-velocity streak, while the modes shown in figures $11(d)$ and $11(e)$, which are sinuous in nature, are associated with disturbances growing in different regions of the low-velocity streak and therefore have considerably lower phase speeds than the shear layer modes. The growth-rate obtained from the BiGlobal eigenvalue problem for the dominant varicose mode is $-\alpha_{i}=0.149$, while the NS and PSE-3D results give respectively $\sigma_{\mathscr{E}}=0.169$ and $\sigma_{K}=0.167$ for the same mode. A disagreement between NS and BiGlobal results was expected given the degree of non-parallelism of the base flow reported in $\$ 4.1$. On the other hand, the agreement between NS and PSE-3D is remarkable.

Difficulties in recovering accurate amplification rates from BiGlobal stability results were also reported by Bonfigli \& Kloker (2007) while studying the secondary 


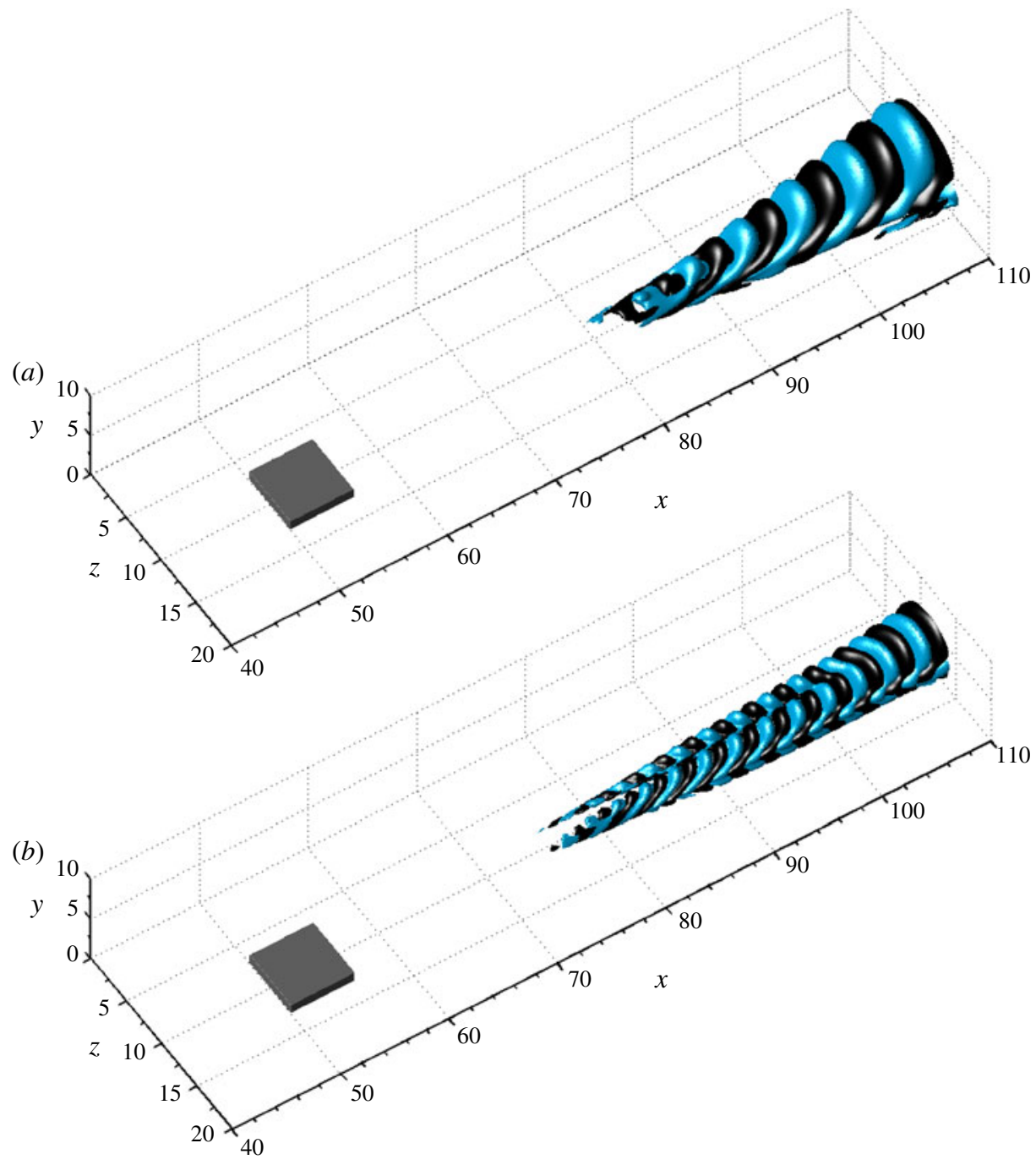

FIGURE 10. (Colour online) Isosurfaces of $\operatorname{Re}\{\hat{v}\}$ (real part of the Fourier-transformed $v$ velocity) showing the three-dimensional structure of $(a) F=0.14$, varicose mode $(\operatorname{Re}\{\hat{v}\}=$ $\left.\pm 5 \times 10^{-4}\right)$ and $(b) F=0.24$, sinuous mode $\left(\operatorname{Re}\{\hat{v}\}= \pm 5 \times 10^{-5}\right)$. Black for positive and grey (blue online) for negative isosurfaces.

instability of cross-flow vortices in an incompressible flow. On the other hand, they show that a BiGlobal stability analysis can give reliable results in terms of mode eigenfunctions and dispersion relation. Here, the $u$-, $v$-, and $w$-velocity amplitude functions of the dominant modes obtained from the BiGlobal eigenvalue analysis are compared with those obtained from the NS simulations in figure 12 for $F=0.08$ and $F=0.26$. It can be seen that the two results are in excellent qualitative agreement. The varicose mode, which dominates the wake instability for $F=0.08$, grows in the three-dimensional shear layer bounding the low-velocity streak and is characterized by maximum $w^{\prime}$ disturbance amplitudes in the lateral regions of the shear layer and $v^{\prime}$ disturbances growing over the whole roughness wake. Note that for the frequency 

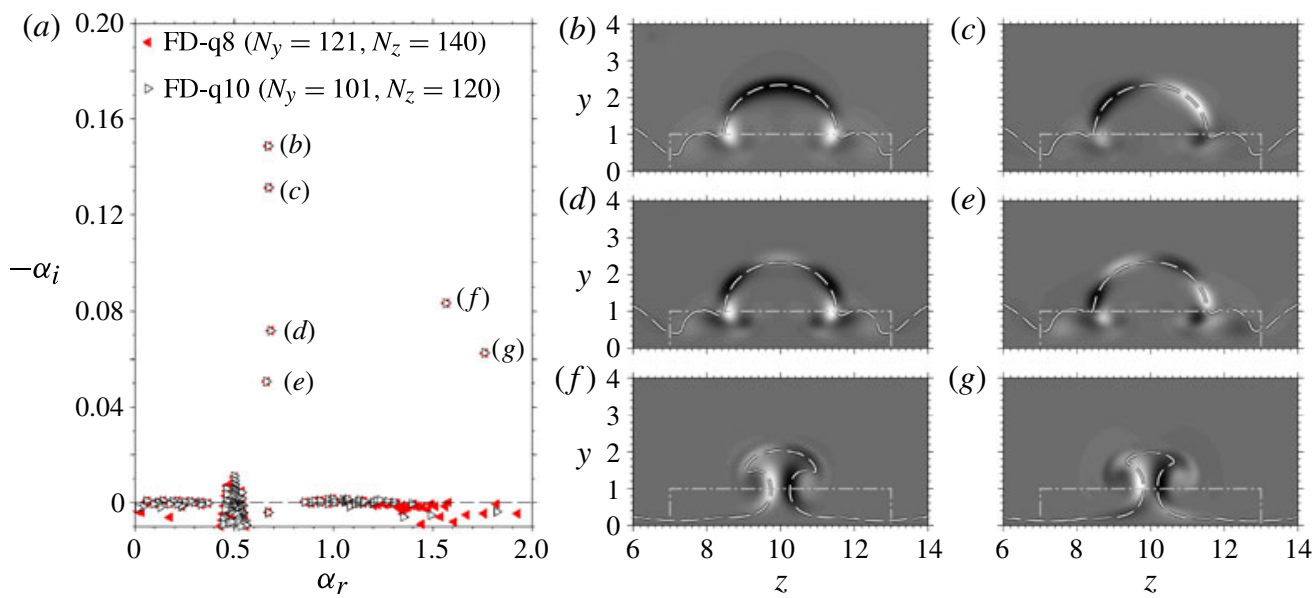

FIGURE 11. (Colour online) Spatial BiGlobal spectrum $(a)$, with letters in parentheses referring to subsequent figures, and contours of the real part of the temperature eigenfunctions (white for negative and black for positive) for some of the most unstable modes $(b-e)$. The dashed lines indicate the critical layers $\left(u_{b}=c_{p h}\right.$, where $c_{p h}$ is the phase speed of the instability modes) and the light-grey dash-dotted line shows the roughness element position. The spectrum and eigenfunctions were obtained for $F=0.08$ at $x=93.66$.

(a)
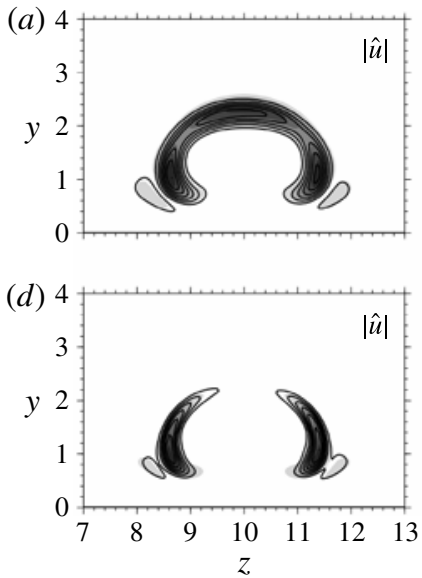

(b)

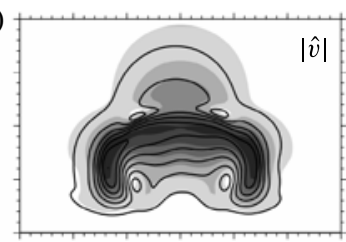

(e)

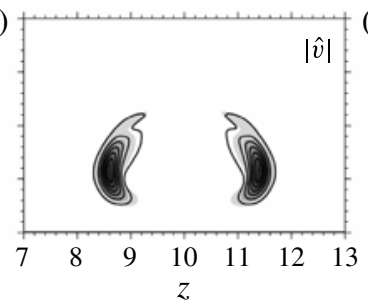

(c)
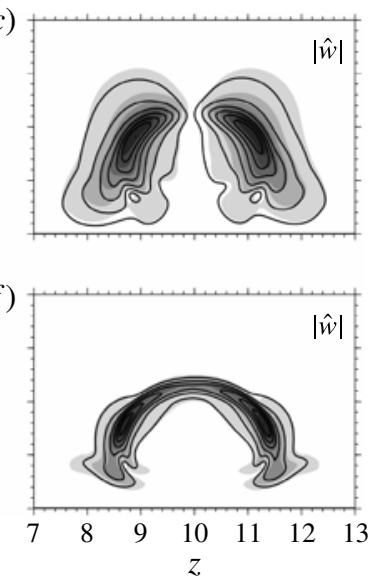

FIGURE 12. Mode shape comparison between Navier-Stokes (black contour lines) and BiGlobal stability (shaded contours from white to grey) results for $x=93.66$. The Navier-Stokes and BiGlobal mode amplitudes were normalized with the maximum attained over $y$ and $z$ and plotted at the same contour levels, eight equally spaced contours from 0 to 1 . $(a-c) F=0.08$ (varicose mode); $(d-f) F=0.26$ (sinuous mode).

considered there is a non-negligible contribution of the sinuous mode to the NS amplitude functions, which leads a slight asymmetry. On the other hand, for $F=0.26$ both the BiGlobal stability and the NS results indicate that the only unstable mode is sinuous and grows in the lateral high-shear layers induced at the sides of the low-velocity streak. As shown in figure $12(d-f)$, the sinuous mode is characterized by 


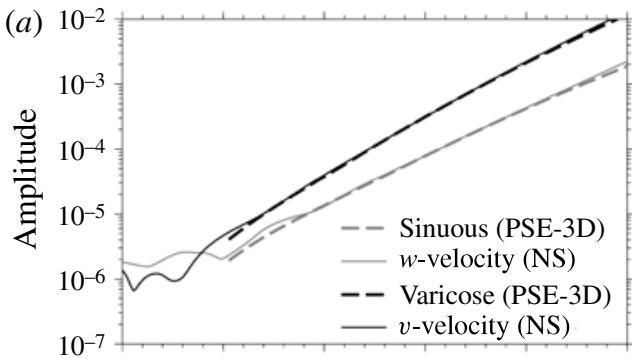

(b)

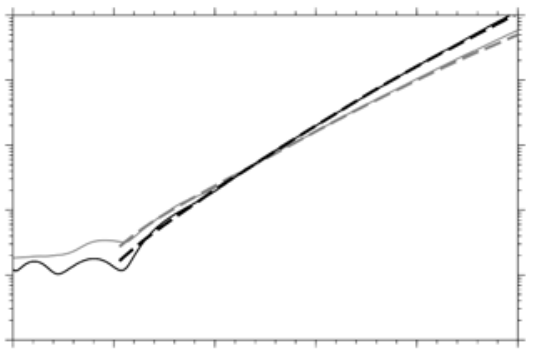

(c)

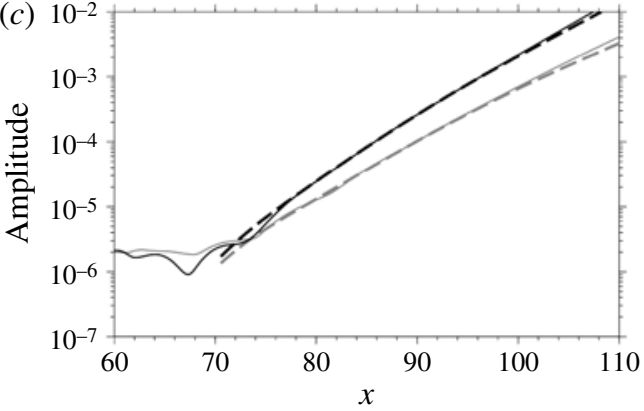

$(d)$

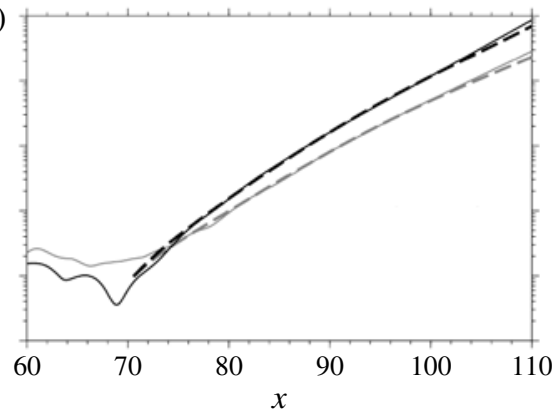

FIGURE 13. Growth of the dominant varicose and sinuous modes; comparison between NS and PSE-3D results. The NS mode amplitudes are calculated as $\int_{0}^{\delta_{99}}|\hat{s}| \mathrm{d} y$ at the roughness mid-plane, where $s=v$ for the varicose mode and $s=w$ for the sinuous mode. The varicose and sinuous PSE-3D mode amplitudes were calculated as $\int_{x} \sigma_{K}(x) \mathrm{d} x$ and normalized to match, respectively, the $v$-velocity and $w$-velocity NS amplitudes at $x=90$ : $(a) F=0.10 ;(b)$ $F=0.12$; (c) $F=0.14 ;(d) F=0.16$.

$v^{\prime}$ disturbances growing in the lateral shear layer regions and $w^{\prime}$ disturbances growing over the whole three-dimensional shear layer.

The structure of the dominant modes taking part in the wake instability can be used to estimate the growth-rates of the varicose and sinuous modes separately from the NS results, which can then be compared with those obtained from the PSE-3D simulations. For this purpose it is worth noting that growth of $v^{\prime}$ disturbances at the roughness mid-plane reflects solely the growth of the varicose mode, while $w^{\prime}$ disturbances at the same location are only influenced by the sinuous mode development (note that $|\hat{w}|=0$ for the varicose mode and $|\hat{v}|=0$ for the sinuous mode at the roughness mid-plane). Figure 13 gives a comparison of the streamwise variation of the sinuous and varicose mode amplitudes obtained from the NS and PSE-3D simulations for some of the most unstable frequencies. Varicose and sinuous mode amplitudes are obtained respectively as $\int_{0}^{\delta_{99}}|\hat{v}| \mathrm{d} y$ and $\int_{0}^{\delta_{99}}|\hat{w}| \mathrm{d} y$ at $z=10$ (roughness mid-plane) from the NS results, while the PSE-3D mode amplitudes are calculated as $\int_{x} \sigma_{K}(x) \mathrm{d} x$. Again NS and PSE-3D results agree closely and indicate that varicose and sinuous modes are unstable for overlapping frequency ranges. The varicose mode dominates throughout the streamwise extent of the computational domain for the most unstable frequencies. In fact, at $x=90$ the growth-rate of the varicose mode is higher than that of the sinuous mode by $\sim 15 \%$ for $F=0.10$ and $F=0.12,11 \%$ for $F=0.14$ and $8 \%$ for $F=0.16$, while at $x=112$ the growth-rate differences are $\sim 16,19,21$ and $22 \%$, respectively. On average (for $80 \leqslant x \leqslant 116$ ) the most unstable varicose mode grows $\sim 17 \%$ faster than the most unstable sinuous mode $\left(\sigma_{K}=0.18\right.$ for $\left.F=0.14\right)$. 

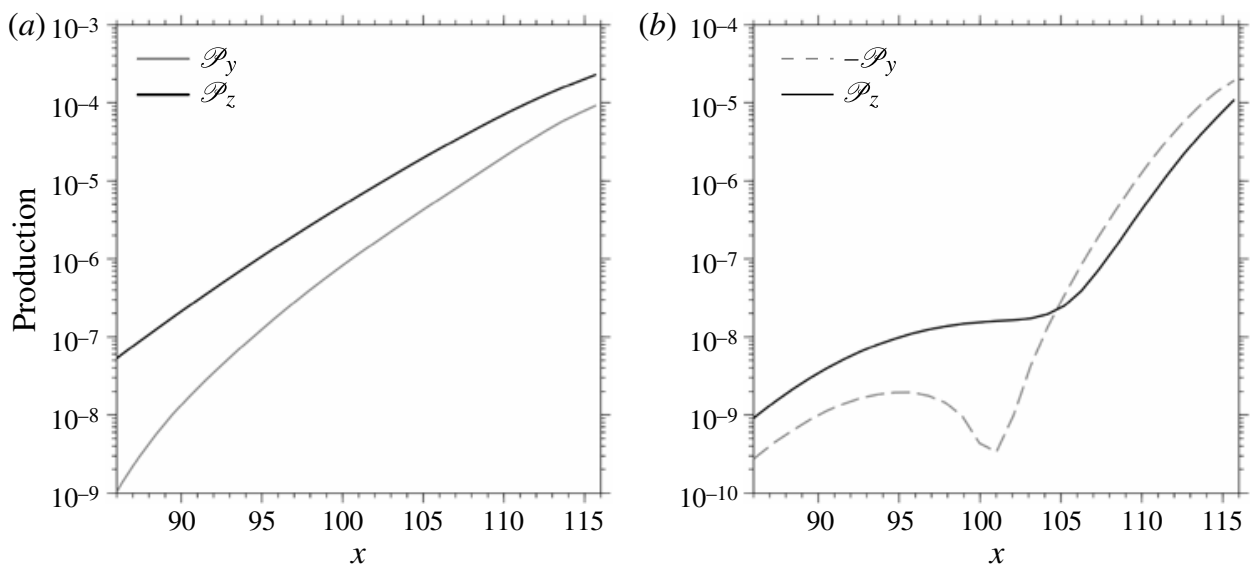

FIGURE 14. Comparison between the production of disturbance kinetic energy due to $\partial u_{b} / \partial y$ $\left(\mathscr{P}_{y}\right)$ and $\partial u_{b} / \partial z\left(\mathscr{P}_{z}\right)$ base flow velocity gradients for $(a)$ the dominant varicose mode at $F=0.08$ and $(b)$ the dominant sinuous mode at $F=0.24$.

These results are in agreement with the BiGlobal stability and experimental results of Choudhari et al. (2010) and Kegerise et al. (2012), which suggest that the varicose mode dominates over the sinuous mode for high $R e_{h}$ (arguably $R e_{h} \geqslant 426$ ). However, contrary to what was found by Choudhari et al. (2010), here the difference in growthrates between varicose and sinuous modes increases for increasing $x$-position, at least within the streamwise extent of the computational domain used, which could be due to a number of reasons including the higher $R e_{h}$ considered here, increased compressibility effects at the higher Mach number or a dependence on the shape of the roughness element. Additional parametric studies would be needed to clarify these findings. Using the N-factor concept (Smith \& Gamberoni 1956; Van Ingen 1956) and assuming $N=9$ as the transition criterion, both the NS and PSE-3D results indicate that transition to turbulence occurs within $\sim 40$ roughness heights from the point of excitation of the varicose mode, which can be considered to be positioned immediately downstream of the separation bubble at the back of the roughness element $(x=66)$. Therefore, transition to turbulence occurs $\sim 50$ roughness heights downstream of the roughness trailing edge. As will be shown in $\S 4.3$, this prediction is in agreement with the results obtained for case NL1.0.

As already mentioned, in the experiments of Asai et al. (2002) the varicose streak instability was found to be the consequence of a Kelvin-Helmholtz instability of the wall-normal detached high-shear layer. In order to understand whether this is also the case here, we calculate the two main contributions $\left(\mathscr{P}_{y}\right.$ and $\left.\mathscr{P}_{z}\right)$ to the production of disturbance kinetic energy $(\mathscr{P})$, given by

$$
\mathscr{P}=\overbrace{-|\hat{u}||\hat{v}| \frac{\partial u_{b}}{\partial y}}^{\mathscr{P}_{y}} \overbrace{-|\hat{u}||\hat{w}| \frac{\partial u_{b}}{\partial z}}^{\mathscr{P}_{z}} .
$$

Figures $14(a)$ and $14(b)$ show a comparison of $\mathscr{P}_{y}$ and $\mathscr{P}_{z}$ for $F=0.08$ and $F=0.24$. Although the instability of the wake is dominated by the varicose mode at $F=0.08$, $\mathscr{P}_{z}$ is larger than $\mathscr{P}_{y}$ over the whole streamwise extent of the computational domain. $\mathscr{P}_{z}$ is also dominant for the sinuous mode, for which case $\mathscr{P}_{y}$ is negative. This result 


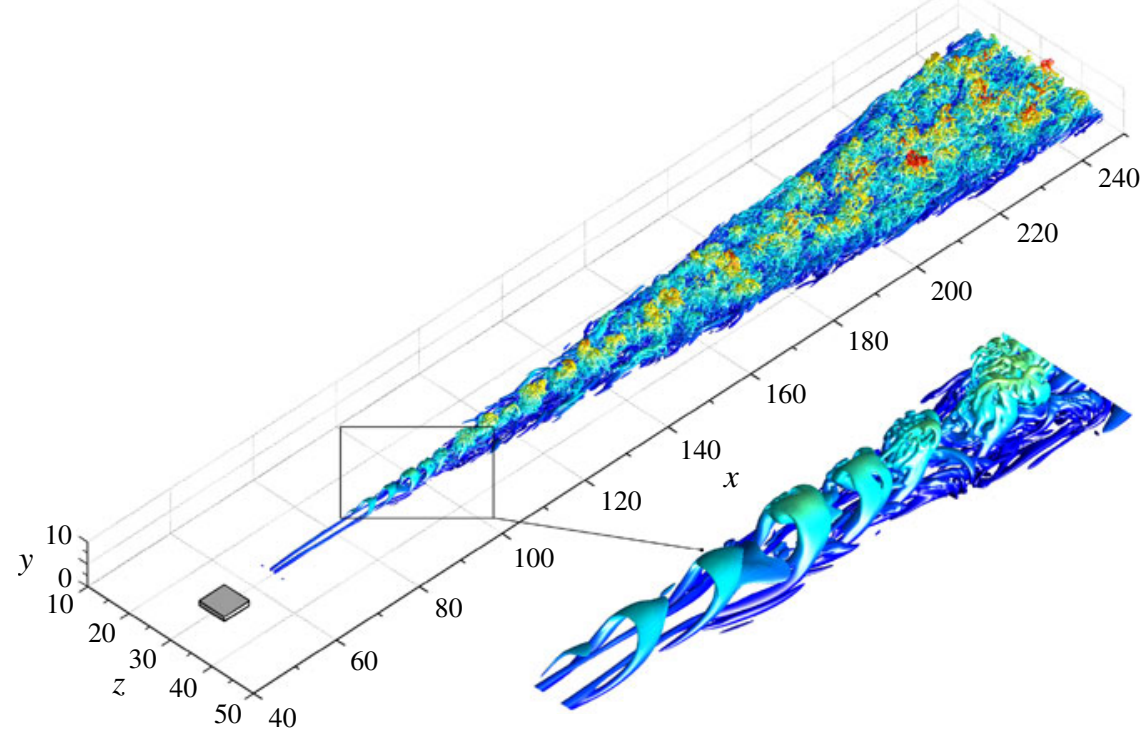

FIGURE 15. (Colour online) Visualization of the turbulent wedge generated downstream of the roughness element after breakdown to turbulence. Isosurfaces of $Q=0.01$ coloured by distance from the wall (blue for low and red for high (online)).

suggests that the sinuous mode is, as expected, the consequence of an instability developing in the lateral high-shear layers. On the other hand, contrary to what would be expected based on the experiments of Asai et al. (2002), it appears that in the case analysed here the varicose mode is the manifestation of an instability of the three-dimensional high-shear layer as a whole rather than a Kelvin-Helmholtz instability of the $\partial u_{b} / \partial y$ part of the shear layer. This observation is also confirmed by careful inspections of the temporal evolution of the flow deformation induced by the disturbances at the frequencies dominated by the varicose mode.

\subsection{Nonlinear breakdown to turbulence}

The analysis provided in the previous sections has shown that, under certain conditions, isolated roughness elements can induce highly unstable wakes which sustain the rapid linear growth of disturbances for a considerable distance downstream of the roughness location. In this section the results obtained for case NL1.0 are discussed to show that, following the linear wake instability, the high-amplitude disturbances present in the roughness wake are able to trigger strong nonlinear interactions, leading to mode saturation and breakdown to turbulence.

Figure 15 shows instantaneous isosurfaces of the second invariant of the velocity gradient tensor ( $Q$-criterion of Chong, Perry \& Cantwell 1990), calculated as

$$
Q=\frac{1}{2}\left[\left(\frac{\partial u_{i}}{\partial x_{i}}\right)^{2}-\frac{\partial u_{i}}{\partial x_{j}} \frac{\partial u_{j}}{\partial x_{i}}\right] .
$$

It can be seen that breakdown to turbulence is initiated by the roll-up of the threedimensional shear layer induced in the roughness wake, leading to the formation of a series of hairpin vortices whose legs connect with the streamwise vortices 

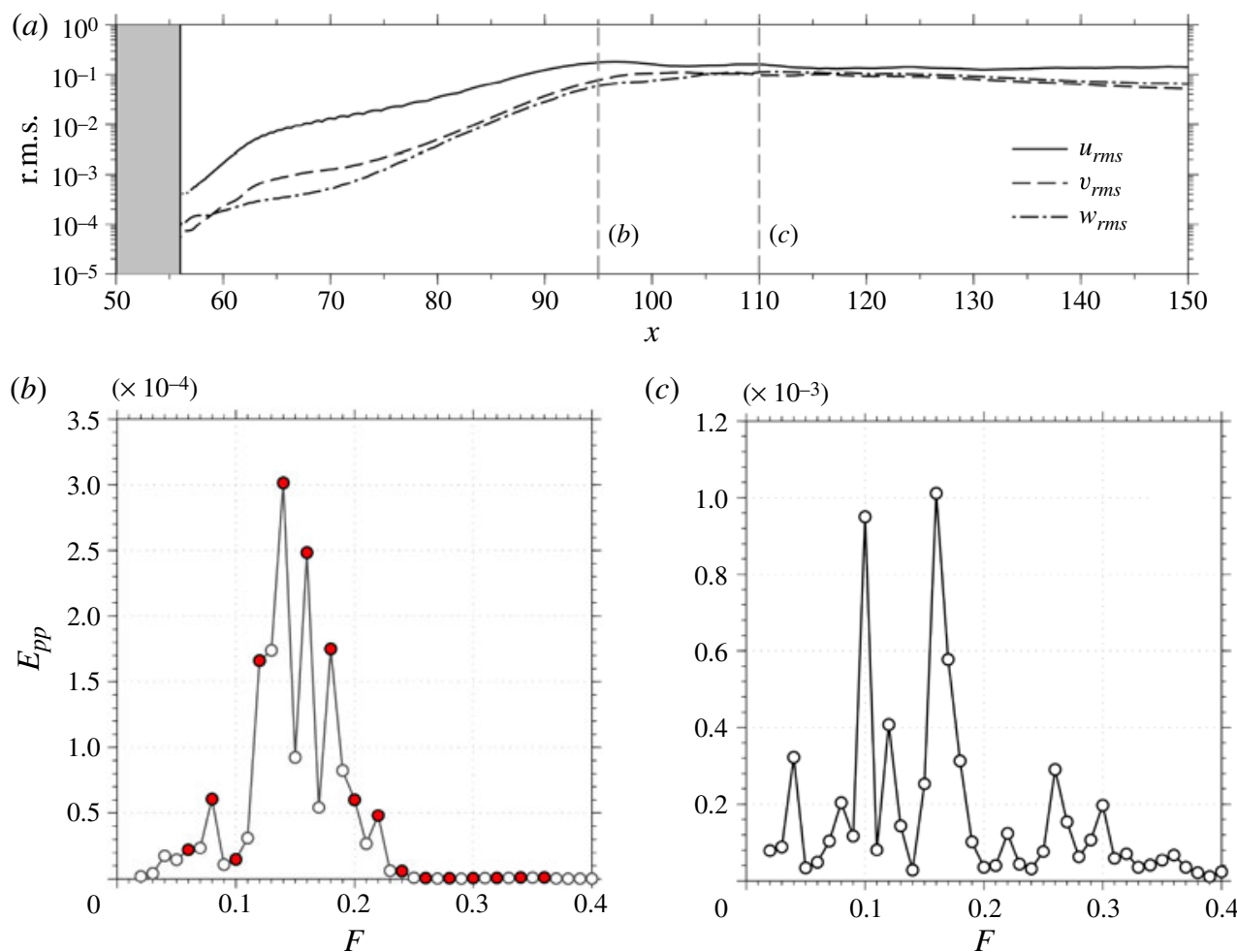

FIGURE 16. (Colour online) Disturbance saturation and nonlinear interactions. (a) Disturbance evolution at the roughness mid-plane $(z=30)$. (b) Wall-pressure spectrum at $x=95, y=0$ and $z=30$. Filled circles indicate the frequencies included in the disturbance forcing signal. (c) Wall-pressure spectrum at $x=110, y=0$ and $z=30$.

induced by the roughness element (see detail). The hairpin structures observed are a consequence of the base flow deformation due to the nonlinear development of the dominant varicose mode. Further downstream the hairpin vortices quickly break down, leading to a turbulent wedge which grows laterally for increasing streamwise distance. Turbulence is continuously generated in the breakdown region (near $x=100$ ) and spreads laterally, also in a continuous fashion.

Figure 16(a) shows the streamwise variation of maximum $u_{r m s}^{\prime}, v_{r m s}^{\prime}$ and $w_{r m s}^{\prime}$ inside the boundary layer at the roughness mid-plane, showing that disturbance saturation occurs starting from about $x=95$. The most amplified frequencies during breakdown to turbulence are shown in figures $16(b)$ and $16(c)$ through energy spectra $\left(E_{p p}=\hat{p}_{w} \hat{p}_{w}^{\dagger}\right)$ obtained from numerical wall-pressure probes located at different positions inside the roughness wake. The spectra were calculated from a total non-dimensional timesample $T_{s}=2100$ using Welch's method (Welch 1967) with 21 periodograms (together with the Hann function). The spectrum in figure $16(b)$ shows that during the initial stages of the nonlinear evolution $(x=95)$ the most amplified frequencies are those found to be dominant during the linear wake instability. As expected, the peak amplitude is found for $F=0.14$, which corresponds to the most unstable wake mode (primary wake instability), identified in $\$ 4.2$ to be of the varicose type. The high receptivity of $F=0.08$, already shown in figure $9(a)$, is also captured in figure $16(a)$ 


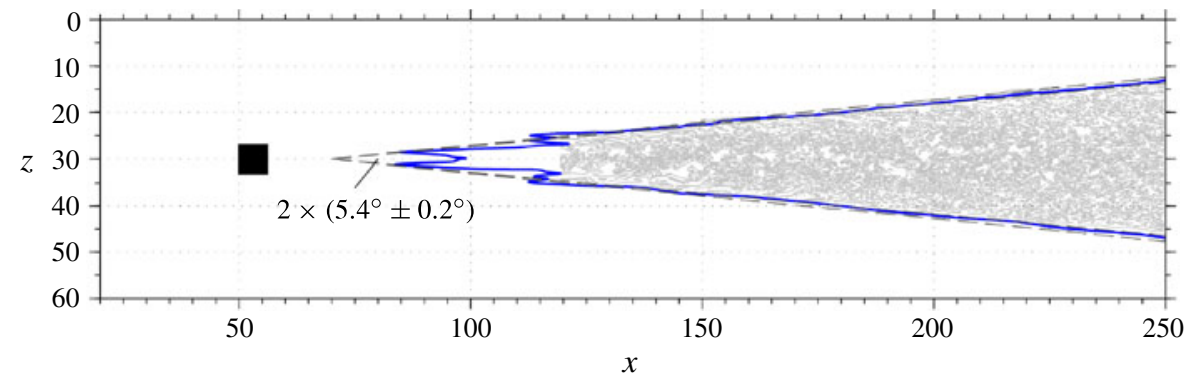

FIGURE 17. (Colour online) Measuring the lateral spreading of the turbulent wedge. Light grey contour lines show regions of instantaneous $\left|\omega_{y}\right|=0.13$, while solid black (blue online) contour line shows TKE $=0.075$, both for $y=1.2$.

as a local energy peak. The spiky appearance of the spectrum is due to the fact that nonlinear interactions are weak at the streamwise position considered, hence the amplitudes of the frequencies not directly excited by the disturbance forcing tend to remain low. Figure 16(c) shows that further downstream $(x=110)$ the frequencies involved in the linear instability of the roughness wake have reached saturation and the spectrum starts to fill up. In particular, peaks can be seen at $F=0.04,0.26$ and 0.30 as a consequence of the sum and difference nonlinear interactions of $F=0.10,0.12$, 0.14 and 0.16 .

The turbulence generated in the breakdown region spreads laterally at a rate which can be quantified by measuring the half-angle of the turbulent wedge developing downstream of the roughness element. Here the half-angle is estimated in two different ways. The first method entails visually measuring the lateral growth of a region of $\mathrm{TKE} \geqslant 0.075$ in a plan view at $y=1.2$ (where TKE is maximum) while the second method looks at the lateral spreading of instantaneous plots of $\left|\omega_{y}\right|=0.13$. The TKE and $\left|\omega_{y}\right|$ cut-off values were chosen because they are representative of the values attained in the core of the turbulent wedge; the former was calculated as an average between minimum and maximum TKE attained at $x=240$, while the latter was obtained as an average between the maximum and minimum (over the $z$-direction) mean $\left|\omega_{y}\right|$, calculated along the $x$-direction for $150 \leqslant x \leqslant 250$. The two procedures are shown in figure 17. It can be seen that both the TKE and the $\left|\omega_{y}\right|$ methods lead to an estimated turbulent wedge half-angle of $\alpha=5.4^{\circ} \pm 0.2^{\circ}$, which lies within the interval derived by Fischer (1972) from experimental data reporting the spreading half-angle of compressible turbulent spots, and is in agreement with more recent DNS and experimental results (Fiala et al. 2006; Krishnan \& Sandham 2006; Redford, Sandham \& Roberts 2012). Assuming that the turbulence spreads laterally starting from a point source, it is also possible to obtain an estimate of the virtual origin of the turbulent boundary layer. As shown in figure 17, the virtual origin is located at about $x=70$ for the case analysed here.

Figure 18(a) shows the streamwise variation of the time-averaged skin friction coefficient at three positions across the span, namely $z=27, z=30$ (the mid-span location) and $z=33$, chosen here to represent the transitional flow behaviour at the centre $(z=30)$ and at the sides $(z=27$ and $z=33)$ of the roughness wake. Upstream of the region of influence of the roughness element $(x<30)$, the skin friction value of the laminar boundary layer agrees well with Eckert's compressible laminar flow correlation (Eckert 1955). Downstream of the roughness element, at the mid-span 

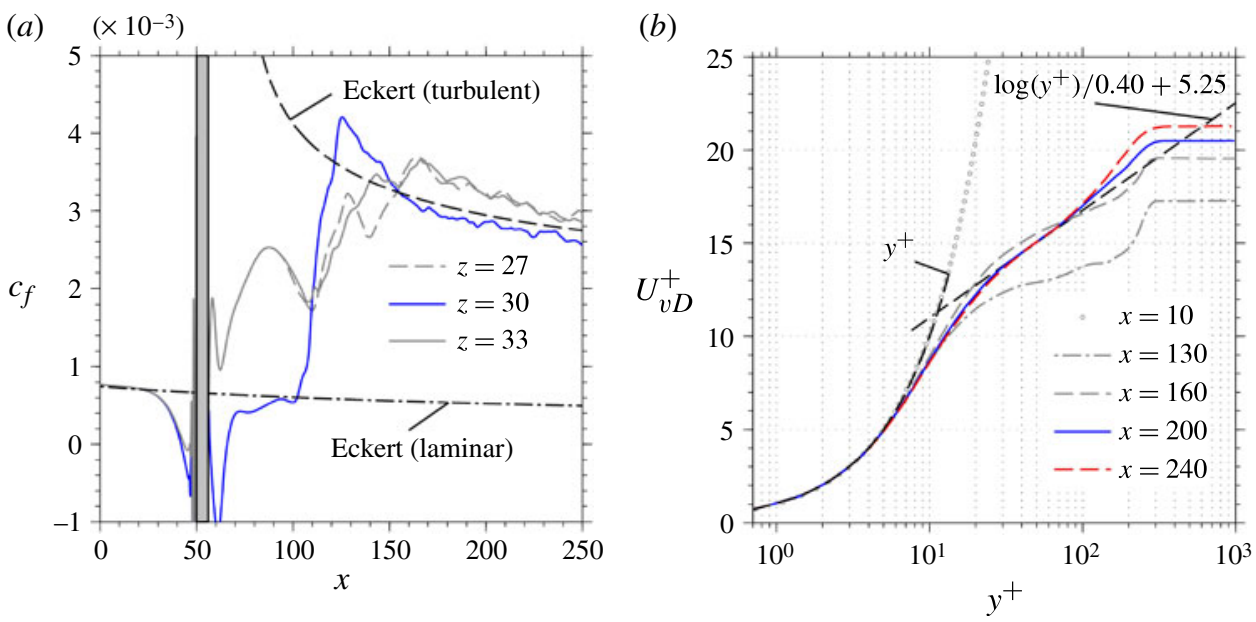

FIGURE 18. (Colour online) Time-averaged boundary layer behaviour. (a) Skin friction variation along the streamwise direction at three different positions across the span. The origin of the turbulent boundary layer for Eckert's skin friction correlation is at $x=70$, while for the laminar correlation the origin is at the flat-plate leading edge. (b) Van Driest scaled mean velocity profiles at different streamwise positions.

location the skin friction rises sharply, starting from $x \approx 100$ due to the breakdown to turbulence; it peaks at $x \approx 125$ and decays further downstream as the turbulent boundary layer grows. At $z=27$ and $z=33$ breakdown to turbulence leads to a gradual skin friction increase starting from $x \approx 110$. The $c_{f}$ values attained at these two lateral wake positions after breakdown to turbulence are higher than those obtained at the roughness mid-plane, which indicates that the effect of the streamwise vortices on the mean flow persists after transition. Interestingly, the measured skin friction and Eckert's turbulent correlation (Eckert 1955) agree well when setting the correlation origin to coincide with the virtual origin of the turbulent wedge measured as in figure 17. Figure 18(b) shows the van Driest scaled mean-velocity profiles at different streamwise locations as a function of wall units $\left(y^{+}\right)$at the roughness mid-plane, showing the boundary layer evolution from the laminar to the turbulent states. The van Driest velocity is calculated using non-dimensional variables as

$$
U_{v D}^{+}=\int_{0}^{u^{+}} \sqrt{\frac{\bar{\rho}}{\bar{\rho}_{w}}} \mathrm{~d} u^{+},
$$

where $u^{+}=\bar{u} / u_{\tau}, \quad u_{\tau}=\left(\bar{v}_{w} / \operatorname{Re}_{\delta_{0}^{*}}[\partial \bar{u} / \partial y]_{w}\right)^{1 / 2}$ is the friction velocity, $v$ is the kinematic viscosity and overbars denote time-averages. The wall units are defined as $y^{+}=y u_{\tau} R e_{\delta_{0}^{*}} / \bar{\nu}_{w}$. A comparison of the mean-velocity profiles in figure $18(b)$ reveals that a fully developed inner layer is established only starting from about $x=200$, while the outer layer is still not fully developed by the end of the computational domain.

Finally, the prediction of transition location made in $\S 4.2$ using the N-factor concept (with $N=9$ ) is compared with the DNS result of case NL1.0. Although the first signs of breakdown to turbulence appear before the skin friction departs from its laminar value (see the formation of the hairpin vortices in figure 15), here we take 
the transition location to coincide with the initial rise in skin friction. This occurs at $x_{t r}=100-110$, in a region where all the disturbances are nonlinearly saturated. Hence, transition to turbulence happens $\sim 44-54$ roughness heights downstream of the roughness trailing edge, in agreement with the $\mathrm{N}$-factor prediction made in $\S 4.2$.

\section{Conclusions}

The laminar-turbulent transition induced by a sharp-edged quadrilateral isolated roughness element at Mach number $M=2.5$ has been thoroughly investigated. The study focused on both the linear instability of the roughness-modified steady base flow and the subsequent nonlinear breakdown to turbulence. The main contributions of the present work are an in-depth analysis of the linear instability of the wake generated behind the roughness element and a cross-validation of full Navier-Stokes, spatial BiGlobal stability and PSE-3D simulations. The latter two analyses were found to agree very well with the Navier-Stokes results. The application of these different methods to the same problem allows the effect of multiple modes and flow nonparallelism to be separated out. PSE-3D simulations were performed here for the first time in the context of compressible flows.

The results show that the base flow changes introduced by the roughness elements can lead to a drastic modification of the stability characteristics of the flow depending on the roughness height considered. Of particular importance is the generation of pairs of counter-rotating streamwise vortices, which, through the lift-up of low-momentum fluid from the near-wall region, give rise to a low-velocity streak surrounded by regions of high wall-normal and lateral shear, forming the roughness wake. While a small roughness element with height over boundary layer thickness ratio of $h^{*} / \delta_{99}=0.22\left(R e_{h}=170\right)$ does not lead to an acceleration of the laminar-turbulent transition process, a roughness element with height $h^{*} / \delta_{99}=0.44\left(R e_{h}=791\right)$ gives rise to a highly unstable wake, where instability modes grow for all frequencies of the forcing. Note that $\delta_{99}$ is taken at the streamwise location of the roughness element in the surrounding undisturbed boundary layer. The spatial BiGlobal stability results show that at least six different modes may grow in the wake of the roughness element. Four of these modes are manifestations of the instability of the three-dimensional shear layer bounding the low-velocity streak, while the remaining two modes reflect disturbances growing in different parts of the streak itself. The two most unstable modes belong to the family of modes developing in the three-dimensional shear layer and are associated with characteristic varicose (symmetric $u^{\prime}$ about the roughness midplane) and sinuous (anti-symmetric $u^{\prime}$ about the roughness mid-plane) deformations of the low-velocity streak. The dominant varicose mode differs from that reported in the experiments of Asai et al. (2002) in that it is a consequence of an instability of the three-dimensional shear layer as a whole rather than a Kelvin-Helmholtz instability of its $\partial u_{b} / \partial y$ part. Both varicose and sinuous modes are most unstable for a non-dimensional frequency $F=0.14$. The varicose mode grows on average $\sim 17 \%$ faster than the sinuous mode, in qualitative agreement with the findings of Choudhari et al. (2010) and Kegerise et al. (2012), and reaches $N=9$ within $\sim 50$ roughness heights from the roughness element trailing edge. The NS results also indicate that when varicose and sinuous modes present similar amplitudes, the total disturbance energy may grow faster that the most unstable mode, as a consequence of the linear superposition of the two modes. This effect was found to be limited for the cases analysed here but it may have wider significance under different conditions. The results obtained from the NS simulations are found to be in excellent agreement 
with those obtained from the stability analyses. The shape functions and growth-rates of the most unstable modes extracted from the NS data are in close agreement with the two-dimensional eigenfunctions obtained from the BiGlobal stability analysis and the growth-rates obtained from the PSE-3D simulations, respectively. Results from an additional DNS show that, following the initial linear instability of the roughness wake, nonlinear interactions lead quickly to mode saturation and breakdown to turbulence and to the generation of a wedge of turbulent flow behind the roughness element. For increasing streamwise distance, the turbulent wedge spreads laterally at a rate similar to that observed in the case of compressible turbulent spots for the same Mach number.

\section{Acknowledgements}

N.D.T. and N.D.S. would like to acknowledge the financial support of the EU through the FP-7 LAPCAT II Project and the computational time on the Hector supercomputer provided by the UK Turbulence Consortium (EPSRC grant EP/G069581/1). The work of P.P. and V.T. was supported by the US Air Force Office of Scientific Research, Air Force Material Command, USAF, under grant number FA8655-12-1-2004. The US Government is authorized to reproduce and distribute reprints for Governmental purpose notwithstanding any copyright notation thereon. The authors would like to thank Professor W. Saric for helpful discussions regarding the definition of the non-dimensional frequency $F$. Valuable comments and suggestions from the anonymous referees are also gratefully acknowledged.

\section{REFERENCES}

Alfredsson, P. H. \& Matsubara, M. 1996 Streaky structures in transition. In Transitional Boundary Layers in Aeronautics (ed. R. Henkes \& J. van Ingen), pp. 374-386. Elsevier.

Amestoy, P. R., Duff, I. S., Koster, J. \& L'Excellent, J.-Y. 2001 A fully asynchronous multifrontal solver using distributed dynamic scheduling. SIAM J. Matrix Anal. Applics 1, $15-41$.

Amestoy, P. R., Guermouche, A., L’Excellent, J.-Y. \& Pralet, S. 2006 Hybrid scheduling for the parallel solution of linear systems. Parallel Comput. 2, 136-156.

Andersson, P., Berggren, M. \& Henningson, D. S. 1999 Optimal disturbances and bypass transition in boundary layers. Phys. Fluids 11 (1), 134-150.

Andersson, P., Brandt, L., Bottaro, A. \& Henningson, D. S. 2001 On the breakdown of boundary layer streaks. J. Fluid Mech. 428, 29-60.

Asai, M., Minagawa, M. \& Nishioka, M. 2002 The instability and breakdown of a near-wall low-speed streak. J. Fluid Mech. 455, 289-314.

BALAKUMAR, P. 2008 Boundary layer receptivity due to roughness and freestream sound for supersonic flows over axisymmetric cones. AIAA Paper 2008-4399.

Bernardini, M., Pirozzoli, S. \& Orlandi, P. 2012 Compressibility effects on roughnessinduced boundary layer transition. Intl J. Heat Fluid Flow 35, 45-51.

Bertolotti, F. P. \& Herbert, T. 1991 Analysis of the linear stability of compressible boundary layers using the PSE. Theor. Comput. Fluid Dyn. 3 (2), 117-124.

Bertolotti, F. P., Herbert, T. \& Spalart, P. 1992 Linear and nonlinear stability of the Blasius boundary layer. J. Fluid Mech. 242, 441-474.

Bonfigli, G. \& KLOKER, M. 2007 Secondary instability of crossflow vortices: validation of the stability theory by direct numerical simulation. J. Fluid Mech. 583, 229-272.

BRANDT, L. \& DE LANGE, H. C. 2008 Streak interactions and breakdown in boundary layer flows. Phys. Fluids 20, 024107.

Bridges, T. J. \& MorRis, P. J. 1984 Differential eigenvalue problems in which the parameter appears nonlinearly. J. Comput. Phys. 55, 437-460. 
Broadhurst, M. \& ShERwIN, S. 2008 The parabolised stability equations for 3D-flows: implementation and numerical stability. Appl. Numer. Maths 58 (7), 1017-1029.

Broadhurst, M., Theofilis, V. \& Sherwin, S. 2006 Spectral element stability analysis of vortical flows. In IUTAM Symposium on Laminar-Turbulent Transition (ed. R. Govindarajan), pp. 153-158. Springer.

Carpenter, M. H., Nordstrom, J. \& Gottlieb, D. 1999 A stable and conservative interface treatment of arbitrary spatial accuracy. J. Comput. Phys. 148, 341-365.

Chang, C. L., Malik, M. R., Erlebacher, G. \& Hussaini, M. Y. 1991 Compressible stability of growing boundary layers using parabolized stability equations. AIAA Paper 91-1636.

Chong, M. S., Perry, A. E. \& Cantwell, B. J. 1990 A general classification of three-dimensional flow fields. Phys. Fluids A 2 (5), 765-777.

Choudhari, M., Li, F., Chang, C. L., Norris, A. \& Edwards, J. 2013 Wake instabilities behind discrete roughness elements in high speed boundary layers. AIAA Paper 2013-0081.

Choudhari, M., Li, F., Minwei, W., Chang, C. L. \& Edwards, J. 2010 Laminar-turbulent transition behind discrete roughness elements in a high-speed boundary layer. AIAA Paper 2010-1575.

Choudhari, M. \& Street, C. L. 1992 A finite Reynolds-number approach for the prediction of boundary-layer receptivity in localized regions. Phys. Fluids A 4 (11), 2495-2514.

Corke, T. C., BAR-Server, A. \& MORKovin, M. V. 1986 Experiments on transition enhancement by distributed roughness. Phys. Fluids 29 (10), 3199-3213.

Crouch, J. D. 1992 Localized receptivity of boundary layers. Phys. Fluids A 4 (7), 1408-1414.

De Tullio, N. \& Sandham, N. D. 2010 Direct numerical simulation of breakdown to turbulence in a Mach 6 boundary layer over a porous surface. Phys. Fluids 22, 094105.

De Tullio, N. \& Sandham, N. D. 2012 Direct numerical simulations of roughness receptivity and transitional shock-wave/boundary-layer interactions. RTO-MP-AVT-200. Art. 22, NATO.

ECKERT, E. R. G. 1955 Engineering relations for friction and heat transfer to surfaces in high velocity flow. J. Aero. Sci. 22 (8), 585-587.

Fiala, A., Hillier, R., Mallinson, S. G. \& Wijesinghe, H. S. 2006 Heat transfer measurement of turbulent spots in a hypersonic blunt-body boundary layer. J. Fluid Mech. 555, 81-111.

FISCHER, M. C. 1972 Spreading of a turbulent disturbance. AIAA J. 10 (7), 957-959.

FUJII, K. 2006 Experiment of the two-dimensional roughness effect on hypersonic boundary-layer transition. J. Spacecr. Rockets $\mathbf{4 3}$ (4), 731-738.

Galionis, I. \& Hall, P. 2005 Spatial stability of the incompressible corner flow. Theor. Comp. Fluid Dyn. 19, 77-113.

Gaster, M., Grosch, C. E. \& Jackson, T. L. 1994 The velocity field created by a shallow bump in a boundary layer. Phys. Fluids 6 (9), 3079-3085.

Goldstein, M. E. 1985 Scattering of acoustic waves into Tollmien-Schlichting waves by small streamwise variations in surface geometry. J. Fluid Mech. 154, 509-529.

Groskopf, G., Kloker, M. J. \& MARXEn, O. 2009 Bi-global crossplane stability analysis of high-speed boundary-layer flows with discrete roughness. In IUTAM Symposium on Laminar-Turbulent Transition (ed. D. Henningson \& P. Schlatter), pp. 171-176. Springer.

Herbert, T. 1994 Parabolized stability equations. AGARD Rep. 793. Special Course on Progress in Transition Modelling, pp. 4(1)-4(34).

Herbert, T. 1997 Parabolized stability equations. Annu. Rev. Fluid Mech. 29, 245-283.

Hermanns, M. \& Hernández, J. A. 2008 Stable high-order finite-difference methods based on non-uniform grid point distributions. Intl J. Numer. Meth. Fluids 56, 233-255.

Horvath, T. J., Berri, S. A. \& Merski, N. R. 2004 Hypersonic boundary/shear layer transition for blunt to slender configurations: a NASA Langley experimental perspective. Tech. Rep. RTO-MP-AVT-111 (22), NATO.

Hultgren, L. S. \& Gustavsson, H. 1981 Algebraic growth of disturbances in a laminar boundary layer. Phys. Fluids 24, 1000.

JAcobs, R. G. \& Durbin, P. A. 2001 Simulations of bypass transition. J. Fluid Mech. 428, $185-212$. 
Jones, L. E. 2008 Numerical study of the flow around an airfoil at low Reynolds number. PhD thesis, School of Engineering Sciences, University of Southampton.

Joslin, R. D. \& GrosCH, C. E. 1995 Growth characteristics downstream of a shallow bump: computation and experiment. Phys. Fluids 7 (12), 3042-3047.

Kegerise, M., King, R., Owens, L., Choudhari, M., Li, F., Chang, C. L. \& Norris, A. 2012 An experimental and numerical study of roughness-induced instabilities in a Mach 3.5 boundary layer. RTO-MP-AVT-200. Art. 29, NATO.

Kegerise, M. A., Owens, L. R. \& Rudolf, A. K. 2010 High-speed boundary-layer transition induced by an isolated roughness element. AIAA Paper 2010-4999.

Klebanoff, P. S. \& Tidstrom, K. D. 1972 Mechanism by which a two-dimensional roughness element induces boundary layer transition. Phys. Fluids 15 (7), 1173-1188.

Krishnan, L. \& SAndhaM, N. D. 2006 Effect of Mach number on the structure of turbulent spots. J. Fluid Mech. 566, 225-234.

LANDAHL, M. T. 1975 Wave breakdown and turbulence. SIAM J. Appl. Maths 28 (4), 735-756.

LI, F. \& MALIK, M. R. 1997 Spectral analysis of parabolized stability equations. Comput. Fluids 26 (3), 279-297.

LI, Q. 2003 Numerical study of the Mach number effect in compressible wall-bounded turbulence. $\mathrm{PhD}$ thesis, School of Engineering Sciences, University of Southampton.

MACK, L. M. 1969 Boundary layer stability theory. JPL Report 900-277, Jet Propulsion Laboratory, California Institute of Technology, Pasadena, CA, USA.

MARXen, O., IACCARINO, G. \& ShaQfeH, S. G. 2010 Disturbance evolution in a Mach 4.8 boundary layer with two-dimensional roughness-induced separation and shock. J. Fluid Mech. 648, 435-469.

Mughal, M. S. 2006 Stability analysis of complex wing geometries: parabolised stability equations in generalized non-orthogonal coordinates. AIAA Paper 2006-3222.

MUPPIDI, S. \& MAHESH, K. 2012 Direct numerical simulations of roughness-induced transition in supersonic boundary layers. J. Fluid Mech. 693, 28-56.

Owens, L. R., Kegerise, M. A. \& Wilkinson, S. P. 2011 Off-body boundary-layer measurement techniques development for supersonic low-disturbance flows. AIAA Paper 2011-0284.

Paredes, P., Hermanns, M., Le Clainche, S. \& Theofilis, V. 2013 Order $10^{4}$ speedup in global linear instability analysis using matrix formation. Comput. Meth. Appl. Mech. Engng 253, 287-304.

Paredes, P., Theofilis, V., Rodríguez, D. \& Tendero, J. A. 2011 The PSE-3D instability analysis methodology for flows depending strongly on two and weakly on the third spatial dimension. AIAA Paper 2011-3752.

Pirozzoli, S., Grasso, F. \& GATSKi, T. B. 2004 Direct numerical simulation and analysis of a spatially evolving supersonic turbulent boundary layer at $M=2.5$. Phys. Fluids 16 (3), 530-545.

REDA, D. C. 2002 Review and synthesis of roughness-dominated transition correlations for reentry vehicles. J. Spacecr. Rockets 39 (2), 161-167.

Reddy, S. C., Schmid, P. J., Baggett, J. S. \& Henningson, D. S. 2001 Simulations of bypass transition. J. Fluid Mech. 428, 185-212.

Redford, J. A., SAndham, N. D. \& Roberts, G. T. 2010 Compressibility effects on boundary-layer transition induced by an isolated roughness element. AIAA J. 48 (12), 2818-2830.

Redford, J. A., Sandham, N. D. \& Roberts, G. T. 2012 Numerical simulations of turbulent spots in supersonic boundary layers: effects of Mach number and wall temperature. Prog. Aerosp. Sci. 52, 67-79.

Reshotko, E. 2001 Transient growth: a factor in bypass transition. Phys. Fluids 13 (5), 1067-1075.

Reshotko, E. \& Tumin, A. 2004 Role of transient growth on roughness-induced transition. AIAA J. 42 (4), 766-770.

Rizzetta, D. P. \& Visbal, M. R. 2007 Direct numerical simulations of flow past an array of distributed roughness elements. AIAA J. 45 (8), 1967-1976. 
Ruban, A. I. 1984 On Tollmien-Schlichting wave generation by sound. Izv. Akad. Nauk SSSR Mekh. Zhidk. Gaza 5, 44-52.

SAAD, Y. 1980 Variations of Arnoldi's method for computing eigenelements of large unsymmetric matrices. Linear Algebr. Applics. 34, 269-295.

SAAD, Y. 1994 SPARSKIT: a basic tool kit for sparse matrix computations, version 2, http:// www-users.cs.umn.edu/ saad/software/SPARSKIT/index.html.

Sandham, N. D., LI, Q. \& YeE, H. C. 2002 Entropy splitting for high-order numerical simulation of compressible turbulence. J. Comput. Phys. 178, 307-322.

SCHNEIDER, S. P. 2008 Effects of roughness on hypersonic boundary-layer transition. J. Spacecr. Rockets 45 (2), 193-209.

Smith, A. M. O. \& Gamberoni, N. 1956 Transition, pressure gradient and stability theory. Rep. ES-26388, Douglas Aircraft Co., El Segundo, California.

Swearingen, J. D. \& BlackWElder, R. F. 1987 The growth and breakdown of streamwise vortices in the presence of a wall. J. Fluid Mech. 182, 255-290.

TANI, I. \& SATO, H. 1956 Boundary-layer transition by roughness element. J. Phys. Soc. Japan 11 (12), 1284-1291.

TheofILIs, V. 1995 Spatial stability of incompressible attachment-line flow. Theor. Comp. Fluid Dyn. 7 (3), 159-171.

THEOFILIS, V. 2003 Advances in global linear instability of nonparallel and three-dimensional flows. Prog. Aerosp. Sci. 39 (4), 249-315.

TheofILIs, V. 2011 Global linear instability. Annu. Rev. Fluid Mech. 43, 319-352.

Thomson, K. W. 1987 Time dependent boundary conditions for hyperbolic systems. J. Comput. Phys. 68, 1-24.

Thomson, K. W. 1990 Time-dependent boundary conditions for hyperbolic systems. Part 2. J. Comput. Phys. 89, 439-461.

Tumin, A. \& Reshotko, E. 2005 Receptivity of a boundary-layer flow to a three-dimensional hump at finite Reynolds numbers. Phys. Fluids 17, 094101.

VAN INGEN, J. L. 1956 A suggested semi-empirical method for the calculation of boundary layer transition region. Rep. UTH-74, Department of Aerospace Engineering, Delft University of Technology.

WELCH, P. D. 1967 The use of fast Fourier transform for the estimation of power spectra: a method based on time-averaging over short, modified periodograms. IEEE Trans. Audio Electroacoust. AU-15, 70-73.

Wheaton, B. M. \& Schneider, S. P. 2012 Roughness-induced instability in a hypersonic laminar boundary layer. AIAA J. 5 (6), 1245-1256.

Wheaton, B. M. \& SChNEIDER, S. P. 2013 Instability and transition due to near-critical roughness in a hypersonic laminar boundary layer. AIAA Paper 2013-0084.

White, F. M 2005 Viscous Fluid Flow, 3rd edn. McGraw-Hill.

Wilkinson, J. H. 1965 The Algebraic Eigenvalue Problem. Clarendon.

WraY, A. A. 1990 Minimal storage time advancement schemes for spectral methods. Rep. M.S. 202 A-1, NASA Ames Research Centre.

WU, X. \& CHOUdhaRI, M. 2003 Linear and nonlinear instabilities of a Blasius boundary layer perturbed by streamwise vortices. Part 2 . Intermittent instability induced by long-wavelength Klebanoff modes. J. Fluid Mech. 483, 249-286.

Yao, Y., Shang, Z., Castagna, J., Johnstone, R., Jones, L. E., Redford, J. A., Sandberg, R. D., Sandham, N. D., Suponitsky, V. \& De Tullio, N. 2009 Re-engineering a DNS code for high-performance computation of turbulent flows. AIAA Paper 2009-566. 